\title{
Practical Continuously Non-Malleable Randomness Encoders in the Random Oracle Model
}

\author{
Antonio Faonio \\ antonio.faonio@eurecom.fr
}

EURECOM, France

\begin{abstract}
A randomness encoder is a generalization of encoding schemes with an efficient procedure for encoding uniformly random strings. In this paper we continue the study of randomness encoders that additionally have the property of being continuous non-malleable. The beautiful notion of non-malleability for encoding schemes, introduced by Dziembowski, Pietrzak and Wichs (ICS10), states that tampering with the codeword can either keep the encoded message identical or produce an uncorrelated message. Continuous non-malleability extends the security notion to a setting where the adversary can tamper the codeword polynomially many times and where we assume a self-destruction mechanism in place in case of decoding errors. Our contributions are: (1) two practical constructions of continuous non-malleable randomness encoders in the random oracle model, and (2) a new compiler from continuous nonmalleable randomness encoders to continuous non-malleable codes, and (3) a study of lower bounds for continuous non-malleability in the random oracle model.
\end{abstract}

\section{Introduction}

The notion of non-malleable codes, Dziembowski, Pietrzak and Wichs [24], (NMC) has emerged at the intersection between cryptography and information theory. Non-malleable codes allow one to encode messages in such a way that, after malicious tampering, the modified codeword decodes to either the original message, or an unrelated one. Non-malleable codes find applications to cryptography, for example, for protecting arbitrary cryptographic primitives against related-key attacks [24] and commitments (Agrawal et al. [4]). Limitations on the nature of the tampering functions must be imposed, as otherwise NMCs are impossible to achieve [24]. One of the most studied settings for which NMCs are achievable is the splitstate model $[2,12,22]$, see also $[3,17,38,40,41]$ In this model we assume that the codeword is divided into two pieces, and that the tampering functions can alter the two pieces independently. 
Continuous non-malleability. In the definition of non-malleable codes, the property is guaranteed as long as a single tampering function is applied to a target codeword. In particular, no security is guaranteed if an adversary can tamper multiple times with the target codeword. While "one-time" non-malleability is already sufficient in some cases, it comes with some shortcomings, among which, for instance, the fact that in applications, after a decoding takes place, we always need to re-encode the message using fresh randomness; the latter might be problematic, as such a reencoding procedure needs to take place in a tamper-proof environment. Motivated by these limitations,Faust et al. [33] introduced a natural extension of non-malleable codes where the adversary is allowed to tamper a target codeword by specifying polynomially-many tampering functions; As argued in [33], such continuously non-malleable codes allow to overcome several limitations of one-time non-malleable codes, and further led to new applications where continuous non-malleability is essential $[13,15]$. Continuous non-malleability requires a special "self-destruct" capability that instructs the decoding algorithm to always output the symbol $\perp$ (meaning "decoding error") after the first invalid codeword is decoded, otherwise generic attacks are possible $[33,35]$. Faust et al.[33] showed that CNMCs are impossible in the information-theoretic setting, while Ostrovsky et al. [41] showed that CNMCs can be constructed assuming that one-to-one one-way functions exist.

Randomness Encoders. A randomness encoder consists of an encoding procedure which can produce a codeword for a random message and the relative decoding algorithm. Kanukurthi, Obbattu and Sekar [36] introduced the concept of non-malleable randomness encoders (NMREs) as a relaxation of NMCs. As shown by [36], NMREs are already sufficient for many of the applications of NMCs. For example, in the typical application of NMCs to tamper-resilient cryptography, the encoded messages are randomly generated secret keys. Moreover, they gave a construction of a NMC from a NMRE and (one-time) authenticated secret key encryption.

Continuous Non-Malleable Randomness Encoder. Dachman-Soled and Kulkarni [16] considered the natural notion of continuous non-malleable randomness encoders (CNMREs). A CNMREs is a relaxation of the concept of continuous non-malleable codes to the realm of randomness encoders. In particular, they showed a construction of CNMRE in the common-reference string (CRS) model assuming only the existence of injective one-way functions and showed a compiler from CNMRE to CNMC for 1-bit messages. 


\subsection{Our Contributions}

As our main contribution, we present two practical CNMREs in the random oracle model. Our randomness encoders can encode random messages of size $\lambda$ bits, where $\lambda$ is the security parameter. The size of the codewords in the first randomness encoder is approximately $12 \lambda$ bits, and the decoding function computes two cryptographic hash evaluations and an inner product between two vectors in $\mathbb{Z}_{p}^{4}$ for a prime $p \geq 2^{\lambda}$. The second randomness encoder has shorter codewords (the size of the codewords is approximately $8 \lambda$ bits), but it has a more expensive decoding function which consists of four cryptographic hash evaluations and an inner product between two vectors in $\mathbb{Z}_{p}^{4}$. Compared to the state-of-art for CNMC (the construction of Ostrovsky et al. [41]) both our randomness encoders are thousands of times more efficient. Comparing with state-of-art for practical NMC (the construction of Fehr,Karpman and Mennick [34]), our randomness encoders are comparably similarly efficient both in terms of sizes of the codewords and in terms of the computational complexities of the algorithms. (We give more details in the next section.)

As second contribution, we show how to construct CNMCs from $\mathrm{CN}$ MREs, thus extending the result of [36] to the continuous setting. We consider the compiler of Coretti, Faonio and Venturi [14], which compiles a CNMC whose ratio between the messages and the codewords is small (asymptotically zero) to a CNMC where the ratio is $\frac{1}{2}$. Although our compiler and their compiler are similar, their analysis does not apply directly to our setting (we elaborate further in the next section). It is well known that continuous non-malleability is impossible in the split-state model with information-theoretic security [33]. As third contribution, we extend the lower bounds of [33] to the case of continuous non-malleability in the random oracle model. We show that we can have information-theoretic security, as long as the number of random oracle queries made by the adversary is bounded.

\subsection{Technical Overview}

In the continuous non-malleability experiment, the adversary receives two messages $\mu_{0}, \mu_{1}$ and gets oracle access to a target codeword $\left(c_{0}, c_{1}\right)$ for the message $\mu_{b}$ with the goal of guessing the bit $b$. The adversary can submit tampering functions $\left(f_{0}, f_{1}\right)$ receiving back the value $\operatorname{Dec}\left(f_{0}\left(c_{0}\right), f_{1}\left(c_{1}\right)\right)$. If the output of the decoding algorithm is $\perp$ then the adversary loses access to the tampering oracle. In the very same vein, in the continuous non-malleability experiment for randomness encoders, the adversary gets 
input two uniformly random keys $\kappa_{0}$ and $\kappa_{1}$ (one of which is sampled using the randomness encoder) and gets oracle access to a target codeword $\left(c_{0}, c_{1}\right)$. We proceed in two steps to construct our CNMREs. In the first step we reduce continuous non-malleability to leakage resilience. In particular for this step, we first define the notion of noisy leakage-resilient randomness encoders (LRREs, for short), then we show an efficient compiler from LRREs to CNMREs. In the second step, we give constructions of leakage-resilient randomness encoders. In the security game for the notion of LRREs the adversary has access to a leakage oracle to the codeword. The adversary can submit queries of the form $\left(g_{0}, g_{1}\right)$ receiving back the values $g_{0}\left(c_{0}\right), g_{1}\left(c_{1}\right)$. In our definition we consider the so-called noisy-leakage model [5] where the leakage is measured as the drop of min-entropy of the codeword.

The compiler from LRREs to CNMREs is very similar to the original construction of CNMC of [33], and its proof of security follows a proof technique similar to $[14,29,32]$. Our LRREs are inspired by the leakage-resilient storage of Davi, Dziembowski and Venturi [18] based on the inner-product extractor (see also Dziembowski and Faust [21]). In more detail, let $\Pi^{\prime}=\left(\right.$ REncode ${ }^{\prime}$, Dec $\left.^{\prime}\right)$ be a LRRE and let RO be a random oracle, we construct a CNMRE $\Pi=$ (REncode, Dec) where the encoding function samples a codeword $c_{0}^{\prime}, c_{1}^{\prime}$ from REncode ${ }^{\prime}$ and then outputs $\left(c_{0}^{\prime}, h_{1}\right),\left(c_{1}^{\prime}, h_{0}\right)$ where $h_{\beta}=\mathrm{RO}\left(\beta \| c_{\beta}^{\prime}\right)$. The decoding function on input a codeword $\left(c_{0}^{\prime}, h_{1}\right),\left(c_{1}^{\prime}, h_{0}\right)$ first checks that the hash values match, namely that $h_{\beta}=\mathrm{RO}\left(\beta \| c_{\beta}^{\prime}\right)$ for $\beta \in\{0,1\}$, and if so it decodes the codeword using Dec'. The main idea behind the security of the scheme is that if an adversary can tamper, let say $c_{0}^{\prime}$, in a non-trivial way obtaining a value $\tilde{c}_{0}^{\prime}$ then it must already know the tampered value $\tilde{c}_{0}^{\prime}$, as otherwise the adversary would not be able to compute correctly $\mathrm{RO}\left(0 \| \tilde{c}_{0}^{\prime}\right)$. (Recall that the adversary can only tamper $c_{0}^{\prime}$ and $h_{0}$ independently, as they are in two different shares.) The latter implies that the output of the tampering oracle is predictable, and therefore we can simulate it using a leakage function that does not decrease the (average conditional) min-entropy of the target codeword.

The second step is to construct practical LRREs. Our first leakageresilient randomness encoder encodes a random message by sampling two random vectors from a field with large enough cardinality, the decoding function outputs the inner product between the two vectors. Previous works considered the encoding scheme where, on input a message $\mu$, the two vectors were sampled conditioned on their inner product being equal to $\mu$. The proofs of security in the previous works relied on (1) the fact 
that the inner product is a two-source extractor and then (2) a complexity leveraging argument to break the dependence between the two vectors. By downgrading to randomness encoders, our proof of security does not need the complexity leveraging argument. This simple observation allows a significant gain in the concrete parameters of the scheme. The second scheme exploits the power of the random oracle. In fact, instead of sampling two vectors, we could sample two seeds which fed to the random oracle would produce the required vectors. By setting the parameters properly, the second randomness encoder has more compact codewords then our first one.

Compiler from randomness-encoders to codes. Similar to $[1,14,36]$, the idea for our compiler is to encode a random key for an authenticated secret key encryption scheme using a CNMRE and then to encrypt the message we want to encode, thus obtaining a ciphertext $\gamma$. As proposed by [14], we store the resulting ciphertext in both sides of the codeword and check for equality of the two copies of the chipertext when decoding. The proof of security of [14] relies on the leakage resilience of the inner CNMC. We show that leakage resilience is not necessary. In fact, any adversarially generated codeword $\left(\tilde{c}_{0}\left\|\tilde{\gamma}_{0}, \tilde{c}_{1}\right\| \tilde{\gamma}_{1}\right)$ (for the compiled code) that successfully decodes must have $\tilde{\gamma}_{0}=\tilde{\gamma}_{1}$. Our novel idea is to use this correlated information to synchronize the tampering functions performed by the reduction and to extract the adversarially generated ciphertext. In more detail, when reducing to the continuous non-malleability of the CNMRE, we additionally sample two valid codewords for two distinct keys $\kappa_{0}, \kappa_{1}$. Upon a tampering query for the compiled code, suppose that the tampered codeword is $\left(\tilde{c}_{0}\left\|\tilde{\gamma}, \tilde{c}_{1}\right\| \tilde{\gamma}\right)$, we first extract, bit-by-bit, the ciphertext $\tilde{\gamma}$ by sending tampering queries that output either $\kappa_{0}$ or $\kappa_{1}$ according to the bits of $\tilde{\gamma}$, and then we send an extra query that allows to decode $\tilde{c}_{0}, \tilde{c}_{1}$, thus obtaining the secret key for the chipertext $\tilde{\gamma}$.

Lower bounds on continuous non-malleability in the ROM. Very roughly speaking, the proof of the impossibility result of [33] shows that any CNMC musts have (at least) two special codewords. The strategy is to hardwire such codewords in their adversary and to use them to extract, bit-by-bit, all the information about the target codeword. However, in the random oracle model, the codeword space is a random variable that depends on the random oracle. Thus an adversary cannot simply have hardwired these two specials codewords, but it needs first to compute them. In other words, the complexity of the generic attack of [33], in our framework, depends on the random-oracle-query complexity of finding 
such two special codewords. Additionally, we show that, even if these two special codewords do not exist, we can still break continuous nonmalleability when the adversary can query the random oracle enough time and de-randomize the full codeword space.

Lastly, we give a lower bound specific to our CNMRE construction. The number of random oracle queries that an adversary needs in order to break the security of our construction depends both on the random-oracle query complexity of the inner leakage-resilient randomness encoder and on the classical birthday-paradox lower bound.

\subsection{Related Work}

In the Table 1 we compare our results with the most relevant related works. We compare with the work of Kiayias, Liu and Tselekounis [37] (resp. the work of Fehr, Karpman and Mennink [34]) which showed a practical construction of NMC in the CRS model (resp. plain model), the work of Dachman-Soled and Kulkarni [16] which showed a construction of CNMRE in the CRS model and a general compiler from CNMREs to 1-bit messages CNMCs, and the work of Ostrovsky, Persiano, Visconti and Venturi [41] which showed a construction of CNMC in the standard model. The result of [41] makes use of a statistically binding commitment scheme and of the leakage-resilient (one-time) non-malleable code of Aggarwal et al. [3]. While, we could implement very efficiently the former ingredient in the random oracle model (by hashing the message together with some randomness), the latter ingredient is the bottleneck of their construction. In fact, the codeword size needs to be at least $O\left(\lambda^{7}\right)$ to encode a message of size $\lambda$. Without diving into the details, if we don't consider the cryptographic hash computations, for both our and their scheme the computational complexity of the decoding function is at least super-linear in the size of the codeword, which implies that our schemes are asymptotically faster than [41] of at least 7 orders of magnitude. We could obtain such a speed up because our schemes rely on the random-oracle methodology, on the other hand, the scheme of Ostrovsky et al. is in the standard model. We stress that the goal of this paper is, indeed, to construct very efficient schemes which could be already used in practice. Dachman-Soled and Kulkarni [16] give a compiler from CNMRE to CNMC. The idea of their compiler is to sample from the encoding procedure of the CNMRE until we obtain a valid codeword of the message to be encoded. The scheme DK $19_{2}$ in Table 1 is the result of applying their compiler to their scheme DK19. . The codeword size is 


\begin{tabular}{|c|c|c|c|c|c|}
\hline Scheme & Non-Malleability & Codeword Size & Type & Model & Assumption \\
\hline$[16]$ DK19 1 & continuous & $\approx 14 \lambda^{3}$ & $\mathrm{R}$ & $\mathrm{CRS}$ & inj. OWF \\
$\Pi_{1}^{*}$ & continuous & $6 \lambda$ & $\mathrm{R}$ & $\mathrm{ROM}$ & - \\
$\Pi_{2}^{*}$ & continuous & $4 \lambda$ & $\mathrm{R}$ & $\mathrm{ROM}$ & - \\
\hline \hline$[34]$ FKM18 & one-time & $2 \lambda+k$ & $\mathrm{E}$ & - & LR-PRP, RK-PRP \\
{$[37] \mathrm{KLT16}$} & one-time & $9 \lambda+2 \log ^{2} \lambda+k$ & $\mathrm{E}$ & $\mathrm{CRS}$ & Ext-HF \\
{$[41]$ OPVV18 } & continuous & $\Omega\left(\lambda^{6} k\right)$ & $\mathrm{E}$ & - & inj. OWF \\
{$[16] \mathrm{DK} 19_{2}$} & continuous & $\approx 14 \lambda^{2}$ & $\mathrm{E}$ & $\mathrm{CRS}$ & inj. OWF \\
$\Pi_{3}^{*}$ & continuous & $8 \lambda+k$ & $\mathrm{E}$ & ROM & OWF \\
\hline
\end{tabular}

Table 1. Comparison with related work. In the table $\lambda$ is the security parameter and $k$ is the length of the message; $\mathrm{R}$ stands for randomness encoders and $\mathrm{E}$ for encoding schemes; inj. OWF stands for injective one-way functions, Ext-HF stands for extractable hash functions, LR-PRP (resp. RK-PRP) stands for leakage-resilient (resp. related-key secure) pseudorandom permutation. OWF stands for one-way functions.

approx. $14 \lambda^{2}$ while the codeword size of DK19 is approx. $14 \lambda^{3}$. The reason is that the compiler works only for 1-bit messages. This limitation is due to the complexity-leveraging argument needed to prove the security of their compiler and the computational security of their scheme DK19. We notice that a natural extension to multi-bit messages of their compiler would work when applied to our scheme $\Pi_{1}^{*}$ because our scheme is secure against unbounded adversaries in the random oracle model. However, the size of the codeword would have a multiplicative blow up in the security parameter $^{1}$. On the other hand, our compiler has only an additive security loss, thus the resulting scheme is more efficient. Additionally, in terms of assumptions, $\Pi_{3}^{*}$ is computationally secure when $k$, the length of the message, is such that $k>\lambda$, however, it can be information-theoretically secure when $k \leq \lambda / 2$.

Non-malleability in the multi-tampering [11,39] model is related to the notion of continuous non-malleability. In the former notion, the number of tampering queries is a priori bounded, however there is no need for self-destruct mechanisms.

Finally, see the following papers that I co-authored (which are slightly related) that I add here only to increase my H-index on Google Scholar $[6,7,8,9,14,26,27,28,29,30,31,32]$.

\footnotetext{
$\overline{1}$ The proof of security would work through a complexity-leveraging argument.
} 


\section{Preliminaries}

We denote with $\lambda \in \mathbb{N}$ the security parameter. A function $\epsilon: \mathbb{N} \rightarrow[0,1]$ is negligible in the security parameter (or simply negligible) if it vanishes faster than the inverse of any polynomial in $\lambda$.

We recall a standard Lemma from Dodis, Reyzin and Smith [20]:

Lemma 1. Let $A, B, C$ be random variables, then

1. For any $\delta>0$, we have $\mathbb{H}_{\infty}(A \mid B=b)$ is at least $\widetilde{\mathbb{H}}_{\infty}(A \mid B)-\log (1 / \delta)$ with probability at least $1-\delta$ over the choice of $b$.

2. If $B$ has at most $2^{\lambda}$ values then $\widetilde{\mathbb{H}}_{\infty}(A \mid B, C) \geq \widetilde{\mathbb{H}}_{\infty}(A \mid C)-\lambda$.

\subsection{Split-State Codes and Randomness-Encoders in the ROM}

Definition 1 (Split-State Encoding Scheme in the ROM, [14]). Let $k(\lambda)=k \in \mathbb{N}$ and $n(\lambda)=n \in \mathbb{N}$ be functions of the security parameter $\lambda \in \mathbb{N}$. A $(k, n)$-split-state-code is a tuple of algorithms $\Sigma=$ $\left(\mathrm{Enc}^{\mathrm{RO}}, \mathrm{Dec}^{\mathrm{RO}}\right)$ specified as follows: (1) The randomized algorithm $\mathrm{Enc}^{\mathrm{RO}}$ takes as input a value $s \in\{0,1\}^{k}$, and outputs a codeword $\left(c_{0}, c_{1}\right) \in$ $\{0,1\}^{2 n}$; (2) The deterministic decoding algorithm Dec ${ }^{\mathrm{RO}}$ takes as input a codeword $\left(c_{0}, c_{1}\right) \in\{0,1\}^{2 n}$, and outputs a value $s \in\{0,1\}^{k} \cup\{\perp\}$ (where $\perp$ denotes an invalid codeword).

We say that $\Sigma$ satisfies correctness if for all values $s \in\{0,1\}^{k}$, $\mathbb{P}\left[\operatorname{Dec}^{\mathrm{RO}}\left(\operatorname{Enc}^{\mathrm{RO}}(s)\right)=s\right]=1$.

We introduce the notion of split-state randomness-encoders in the ROM.

Definition 2 (Split-State Randomness Encoders in the ROM). Let $n(\lambda)=n \in \mathbb{N}$ be functions of the security parameter $\lambda \in \mathbb{N}$. A $n$-splitstate-randomness-encoder is a tuple of algorithms $\Pi=\left(\mathrm{REncode}^{\mathrm{RO}}, \mathrm{Dec}^{\mathrm{RO}}\right)$ specified as follows: (1) The randomized algorithm $\mathrm{REncode}^{\mathrm{RO}}$ (with the only input the security parameter) outputs a value $\kappa \in\{0,1\}^{\lambda}$ and a codeword $\left(c_{0}, c_{1}\right) \in\{0,1\}^{2 n}$; (2) The deterministic decoding algorithm $\operatorname{Dec}^{\mathrm{RO}}$ takes as input a codeword $\left(c_{0}, c_{1}\right) \in\{0,1\}^{2 n}$, and outputs a value $\kappa \in\{0,1\}^{\lambda} \cup\{\perp\}$ (where $\perp$ denotes an invalid codeword). We say that $\Sigma$ satisfies correctness if for all $\lambda$ the following holds:

$\mathbb{P}\left[\kappa=\kappa^{\prime} \wedge(\kappa, c) \leftarrow \mathrm{REncode}^{\mathrm{RO}}\left(1^{\lambda}\right) \wedge \kappa^{\prime} \leftarrow \operatorname{Dec}^{\mathrm{RO}}(c)\right]=1$.

The contributions of this paper focus on split-state encoding schemes and split-state randomness encoders. To avoid redundancy, we therefore omit the adjective "split-state" whenever it is clear from the context. Many of 
the algorithms described in this paper make use of a random oracle, we avoid to upper script them with the oracle RO whenever it is clear from the context.

\subsection{Continuous non-malleability in the ROM}

An encoding scheme is non-malleable $[24,40]$ if no adversary tampering independently with the two parts of the target encoding $\left(c_{0}, c_{1}\right)$ can generate a modified codeword that decodes to a related value. Continuous non-malleability [33] strengthens this guarantee by allowing to tamper continuously and adaptively with $\left(c_{0}, c_{1}\right)$, until a decoding error occurs, after which the system "self-destructs" and stops answering tampering queries. The self-destruct, that in practice could be implemented via a write-once flag, is strictly necessary (see [35]) for achieving continuous non-malleability whenever no other refresh mechanisms are in place (see $[28,29])$. Because we consider schemes in the random oracle model, we enlarge the class of possible tampering functions considering functions that additionally can query the random oracle RO. Consider the following class of tampering functions parameterized by two values $n(\lambda), q(\lambda)$ :

$$
\mathcal{F}_{n, q}=\left\{\left(f_{0}, f_{1}\right) \mid \forall b: \begin{array}{c}
f_{b}:\{0,1\}^{n(\lambda)} \rightarrow\{0,1\}^{n(\lambda)} \\
f_{b} \text { makes at most } q(\lambda) \text { RO queries }
\end{array}, \lambda \in \mathbb{N}\right\}
$$

Definition 3 (Continuously non-malleable codes and randomness encoders in the ROM). Let $k(\lambda), n(\lambda), q(\lambda), q_{T}(\lambda), q_{\mathrm{RO}}(\lambda) \in \mathbb{N}$ and let $\epsilon(\lambda) \in \mathbb{R}$.

Let $\Sigma=($ Enc, Dec) be a $(k, n)$-encoding scheme. We say that $\Sigma$ is $\left(\epsilon, q, q_{\mathrm{RO}}\right)$-continuously non-malleable code, $\left(\epsilon, q, q_{\mathrm{RO}}\right)$-CNMC for short, if for all messages $\mu_{0}, \mu_{1}$, for all $q_{T}=\operatorname{poly}(\lambda)$, and for all unbounded adversaries A making up to $q_{T}$ tampering oracle queries from the class of tampering functions $\mathcal{F}_{n, q}$ and up to $q_{\mathrm{RO}}$ random oracle queries, we have:

$$
\operatorname{Adv}_{\Sigma, \mathbf{A}}^{\mathrm{cnmc}}(\lambda):=\left|\mathbb{P}\left[\mathbf{G}_{\Sigma, \mathbf{A}}^{\mathrm{cnmc}}\left(\lambda, \mu_{0}, \mu_{1}\right)=1\right]-1 / 2\right| \leq \epsilon(\lambda) .
$$

Let $\Pi=($ REncode, $\mathrm{Dec})$ be a $n$-randomness encoder. We say that $\Pi$ is $\left(\epsilon, q, q_{\mathrm{RO}}\right)$-continuously non-malleable randomness encoder, $\left(\epsilon, q, q_{\mathrm{RO}}\right)$ CNMRE for short, if for all $q_{T}=\operatorname{poly}(\lambda)$, and for all (possibly unbounded) adversaries A making up to $q_{T}$ tampering oracle queries from the class of tampering functions $\mathcal{F}_{n, q}$ and up to $q_{\mathrm{RO}}$ random oracle queries, we have:

$$
\operatorname{Adv}_{I, \mathbf{A}}^{\mathrm{cnmre}}(\lambda):=\left|\mathbb{P}\left[\mathbf{G}_{\Sigma, \mathbf{A}}^{\mathrm{cnmre}}(\lambda)=1\right]-1 / 2\right| \leq \epsilon(\lambda) .
$$




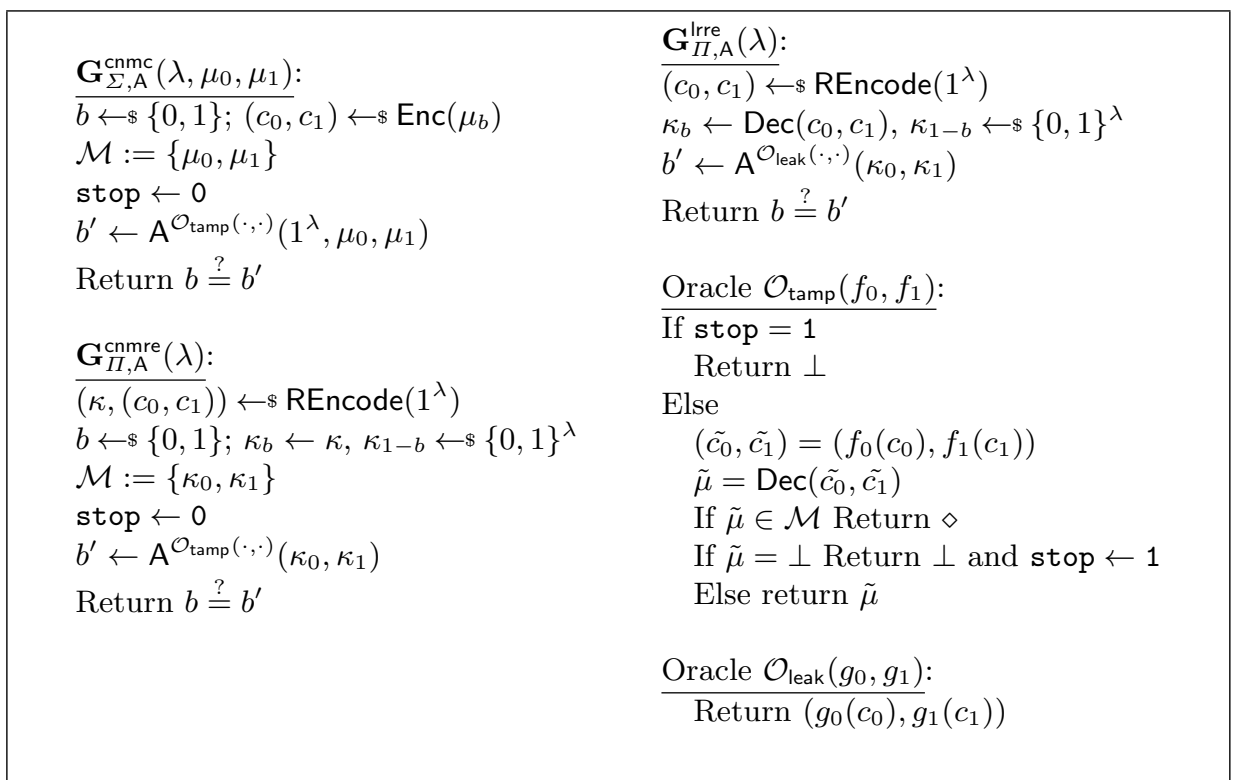

Fig. 1: Experiment defining continuously non-malleable codes and randomnessencoders in the split-state model, and leakage-resilient randomness encoders. The tampering oracle $\mathcal{O}_{\text {tamp }}$ is implicitly parameterized by the flag stop, the codeword $c_{0}, c_{1}$ and the set $\mathcal{M}$. Similarly, the leakage oracle is implicitly parameterized by the codeword $c_{0}, c_{1}$. If $\Pi$ (resp. $\Sigma$ ) is in the random oracle model, then all the procedures and functions (including the adversary $\mathrm{A}$, the leakage functions $g_{0}, g_{1}$ and the tampering functions $\left.f_{0}, f_{1}\right)$ implicitly have oracle access to RO.

The experiments $\mathbf{G}_{\Sigma, \mathbf{A}}^{\mathrm{cnmc}}(\lambda)$ and $\mathbf{G}_{\Sigma, \mathbf{A}}^{\mathrm{cnmc}}\left(\lambda, \mu_{0}, \mu_{1}\right)$ are described in Fig. 1.

Remark 1 (On the choice of $q_{T}$ ). We could prove security of our constructions even when $q_{T}=\Omega\left(2^{\lambda}\right)$. However, in the definitions above we limit the number of tampering queries to be a polynomial in the security parameter. The reason is that for each tampering query there is an associated call to the decoding algorithm of the attacked device. We can assume that the attacked device runs in polynomial time.

\subsection{Noisy-leakage resilient randomness encoders.}

As in previous works $[9,14,29,32]$, we use the notion of admissibility to define noisy-leakage resilience. We extend this notion to the ROM.

Definition 4 (Admissible adversaries for randomness encoders). Let $n(\lambda), \ell(\lambda), q_{\mathrm{RO}}(\lambda) \in \mathbb{N}$ such that $\ell(\lambda) \leq n(\lambda)$, let $\Pi=$ (REncode, Dec) be a $n$-randomness-encoder. An adversary $\mathrm{A}$ is $\left(\ell, q_{\mathrm{RO}}\right)$-admissible if: 
1. it outputs a sequences of leakage queries (chosen adaptively) $\left(g_{0}^{(i)}, g_{1}^{(i)}\right)_{i \in[q]}$ for $q \in \mathbb{N}$, such that for any $i$ the functions $\left(g_{0}^{(i)}, g_{1}^{(i)}\right)$ can make queries to the random oracle,

2. it outputs a sequences of random oracle queries (adaptively) $\left(x_{i}\right)_{i \in\left[q_{\mathrm{RO}}\right]}$, such that:

$\left.\widetilde{\mathbb{H}}_{\infty}\left(c_{\beta} \mid c_{1-\beta}, g_{\beta}^{(1)}\left(c_{\beta}\right), \cdots, g_{\beta}^{(p)}\left(c_{\beta}\right),\left(x_{i}, \mathrm{RO}\left(x_{i}\right)\right)_{i \in\left[q_{\mathrm{RO}}\right]}\right)\right) \geq \widetilde{\mathbb{H}}_{\infty}\left(c_{\beta} \mid c_{1-\beta}, \mathrm{RO}\right)-\ell$

where $\left(c_{0}, c_{1}\right)$ is the joint random variable corresponding to REncode $\left(1^{\lambda}\right)$

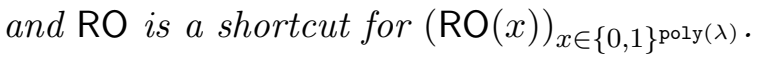

We define the notion of noisy-leakage resilient randomness encoders (LRREs).

Definition 5 (noisy-leakage resilient randomness encoders). Let $n(\lambda), \ell(\lambda), q_{\mathrm{RO}}(\lambda) \in \mathbb{N}$ and $\epsilon(\lambda) \in \mathbb{R}$, and let $\Pi=($ REncode, Dec) be a $n$ randomness encoder. We say that $\Pi$ is $\left(\epsilon, \ell, q_{\mathrm{RO}}\right)$-noisy-leakage resilient randomness encoder in the $R O M,\left(\epsilon, \ell, q_{\mathrm{RO}}\right)-L R R E$ for short, if for all $\left(\ell, q_{\mathrm{RO}}\right)$-admissible adversaries $\mathrm{A}$, we have that:

$$
\operatorname{Adv}_{I I, \mathbf{A}}^{\text {Irs }}(\lambda):=\left|\mathbb{P}\left[\mathbf{G}_{\Sigma, \mathbf{A}}^{\text {Irre }}(\lambda)=1\right]-1 / 2\right| \leq \epsilon(\lambda),
$$

where experiment $\mathbf{G}_{\Sigma, \mathbf{A}}^{\mathrm{Irre}}(\lambda)$ is depicted in Fig. 1.

Remarks on admissibility in the ROM. To obtain our notion of admissibility in the ROM we considered several factors. Below, we clarify our choices:

The leakage functions can make random oracle queries. Beside being natural, this requirement avoids to trivialize our notions of security. In fact, consider the randomness encoder in which the codewords $c$ are random strings in $\{0,1\}^{\ell^{\prime}}$ for a parameter $\ell^{\prime} \in \mathbb{N}$, and where the decoding algorithm simply outputs $\mathrm{RO}(c)$. It is not hard to see that the scheme would be secure against leakage and tampering attacks that cannot make random oracle queries, even if both the tampering and the leakage queries could act over the full codeword (i.e., no split-state). However, this is in stark contrast with what we can achieve in the plain model, where [24] showed that this form of leakage and tamper resilience is indeed impossible. 
Full-knowledge of the random oracle. We condition on the full knowledge of the random oracle RO in the conditional min-entropy in the r.h.s. of Eq. 3. Intuitively, the value on the right hand side measures the leakage resilience of the randomness encoder. Previous works (that did not consider random oracles) $[14,32,9]$ the r.h.s. of the equation above would simply be the conditional average min-entropy of $c_{\beta}$ given $c_{1-\beta}$, namely, the amount of randomness in each side of the codeword without considering the mutual information. We instead consider the conditional average min-entropy of $c_{\beta}$ given $c_{1-\beta}$ and the full description of the random oracle. There are two main reasons: first we do not want to use the random oracle to artificially blow randomness inside the codeword and thus having an artificially big (but useless) leakage parameter $\ell$; second, the two parts of the codeword $c_{0}, c_{1}$ could be one a (randomized) function of the other (let say $c_{0}=f\left(c_{1}\right)$ for some randomized function $f$ that uses the random oracle) in this case we want to preserve the measure $\widetilde{\mathbb{H}}_{\infty}\left(f\left(c_{1}\right) \mid c_{1}\right)$ "as it was in the plain model". Namely, the measure should be a function of the amount of randomness that $f$ uses and not of the randomness that $f$ borrows from the random oracle. By conditioning on the knowledge of the full description of the function RO we, indeed, de-randomize the oracle RO. Informally speaking, we would like to have that for any "real-world instantiation $F$ of the random oracle" the value $\widetilde{\mathbb{H}}_{\infty}\left(f^{F(\cdot)}\left(c_{1}\right) \mid c_{1}\right)$ and the value $\widetilde{\mathbb{H}}_{\infty}\left(f^{\mathrm{RO}(\cdot)}\left(c_{1}\right) \mid c_{1}, \mathrm{RO}\right)$ are the same ${ }^{2}$.

Queries to the random oracle in the view of the adversary. We included only the queries of the adversaries to the random oracle RO in the l.h.s. of Eq. 3, instead of the full description of RO. The reason is that the left hand side of the equation should model the information that the adversary has gathered during the security experiment.

\subsection{Authenticated Encryption}

A secret-key encryption (SKE) scheme is a tuple of algorithms $\Omega:=$ (AEnc, ADec) specified as follows: (1) The randomized algorithm AEnc takes as input a key $\kappa \in\{0,1\}^{\lambda}$, a message $\mu \in\{0,1\}^{k}$, and outputs a ciphertext $\gamma \in\{0,1\}^{m} ;(2)$ The deterministic algorithm ADec takes as input a key $\kappa \in\{0,1\}^{\lambda}$, a ciphertext $\gamma \in\{0,1\}^{m}$, and outputs a value $\mu \in\{0,1\}^{k} \cup\{\perp\}$ (where $\perp$ denotes an invalid ciphertext). The values $k(\lambda), m(\lambda)$ are all polynomials in the security parameter $\lambda \in \mathbb{N}$, and sometimes we call $\Omega$ an $(k, m)$-SKE scheme.

\footnotetext{
2 Notice this is not true in general, as $f$ could be a contrived function that depends of the specification of $F$.
} 


\begin{tabular}{|c|c|}
\hline $\begin{array}{l}\mathrm{G}_{\Omega, \mathrm{A}}^{\text {ind }}(\lambda): \\
b \leftarrow\{0,1\} ; \kappa \leftarrow \$\{0,1\}^{\lambda} \\
\left(\mu_{0}, \mu_{1}, \alpha\right) \leftarrow \$ \mathrm{~A}_{0}\left(1^{\lambda}\right) \\
\gamma \leftarrow \mathrm{AEnc}\left(\kappa, \mu_{b}\right) \\
b^{\prime} \leftarrow \mathrm{A}_{1}(\gamma, \alpha) \\
\text { Return } b=b^{\prime}\end{array}$ & $\begin{array}{l}\mathbf{G}_{\Omega, \mathrm{A}}^{\text {auth }}(\lambda): \\
\kappa \leftarrow\{0,1\}^{\lambda} \\
(\mu, \alpha) \leftarrow \$ \mathrm{~A}_{0}\left(1^{\lambda}\right) \\
\gamma \leftarrow \$ \operatorname{AEnc}(\kappa, \mu) \\
\gamma^{\prime} \leftarrow \$ \mathrm{~A}_{1}(\gamma, \alpha) \\
\text { Return } \gamma^{\prime} \neq \gamma \wedge \operatorname{ADec}\left(\kappa, \gamma^{\prime}\right) \neq \perp\end{array}$ \\
\hline
\end{tabular}

Fig. 2: Experiments defining security of SKE.

We say that $\Omega$ meets correctness if for all $\kappa \in\{0,1\}^{\lambda}$, all messages $\mu \in\{0,1\}^{k}$, we have that $\mathbb{P}[\operatorname{ADec}(\kappa, \operatorname{AEnc}(\kappa, \mu))=\mu]=1$ (the probability is taken over the randomness of $A E n c)$.

Definition 6 (Security of SKE). Let $\Omega=$ (KGen, AEnc, ADec) be a $S K E$ scheme. We say that $\Omega$ is $(\epsilon, \delta)$-secure if the following holds for the games defined in Fig. 2. For all (possibly unbounded) adversaries $\mathrm{A}$ the following advantages $\mathbf{A d v}_{\Omega, \mathbf{A}}^{\text {auth }}(\lambda):=\mathbb{P}\left[\mathbf{G}_{\Omega, \mathbf{A}}^{\text {auth }}(\lambda)=1\right]$ and $\mathbf{A d v}_{\Omega, \mathbf{A}}^{\text {ind }}(\lambda):=$ $\left|\mathbb{P}\left[\mathbf{G}_{\Omega, A}^{\text {ind }}(\lambda)=1\right]-\frac{1}{2}\right|$ are negligible.

Note that since both authenticity and indistinguishable encryption are one-time properties, information-theoretic constructions with such properties exist.

\section{Our Continuous Non-Malleable Randomness Encoder}

Let $\Pi=$ (REncode, Dec) be a $n^{\prime}$-randomness-encoder, and let RO be a random oracle. Consider the following construction of a $n$-randomnessencoder $\Pi^{*}=\left(\right.$ REncode ${ }^{*}$, Dec $\left.{ }^{*}\right)$ where $n:=n^{\prime}+2 \lambda$ :

$\operatorname{REncode}^{*}\left(1^{\lambda}\right)$ : Sample $\kappa,\left(c_{0}, c_{1}\right) \leftarrow \$ \operatorname{REncode}\left(1^{\lambda}\right)$, compute $h_{\beta} \leftarrow \operatorname{RO}\left(\beta \| c_{\beta}\right)$ and set the codeword $c_{\beta}^{*}:=\left(c_{\beta}, h_{1-\beta}\right)$ for $i \in\{0,1\}$. Return $\kappa,\left(c_{0}^{*}, c_{1}^{*}\right)$. $\operatorname{Dec}^{*}\left(c_{0}^{*}, c_{1}^{*}\right)$ : Execute the following steps:

1. For $\beta \in\{0,1\}$, parse $c_{\beta}^{*}$ as $\left(c_{\beta}, h_{1-\beta}\right)$;

2. (Hash Values Check.) If $h_{0} \neq \mathrm{RO}\left(0 \| c_{0}\right)$ or $h_{1} \neq \mathrm{RO}\left(1 \| c_{1}\right)$ output $\perp$;

3. Else output $\operatorname{Dec}\left(c_{0}, c_{1}\right)$.

We give the following definition to simplify the notation in the statement of the theorem.

Definition 7. Let $\Pi$ be a n-randomness encoder, we define with $\alpha_{\Pi}(\lambda):=$ $\min _{\beta}\left\{\mathbb{H}_{\infty}\left(c_{\beta}\right)-\widetilde{\mathbb{H}}_{\infty}\left(c_{\beta} \mid c_{1-\beta}, \mathrm{RO}\right)\right\}$ where $c_{0}, c_{1} \leftarrow$ REncode $\left(1^{\lambda}\right)$. 
Theorem 1 (LRREs $\Rightarrow$ CNMREs in ROM). For any $q_{T}:=q_{T}(\lambda), q:=$ $q(\lambda)$, and for any adversary $\mathrm{A}$ that does up to $q_{T}$ tampering oracle queries from the class of tampering functions $\mathcal{F}_{n, q}$ and up to $q_{\mathrm{RO}}:=q_{\mathrm{RO}}(\lambda)$ random oracle queries there exists a $\left(\ell, q_{\mathrm{RO}}\right)$-admissible adversary $\mathrm{B}$ where $\ell=2 \log q_{\mathrm{RO}}+\log q_{T}$ such that:

$$
\operatorname{Adv}_{\Pi^{*}, \mathrm{~A}}^{\mathrm{cnmre}}(\lambda) \leq \mathbf{A} \mathbf{d} \mathbf{v}_{\Pi, \mathrm{B}}^{\mathrm{Irs}}(\lambda)+\frac{q_{T}}{2^{2 \lambda}}+\frac{\left(q_{\mathrm{RO}}+q \cdot q_{T}\right)^{2}}{2^{2 \lambda}}+\frac{\left(q_{\mathrm{RO}}+q \cdot q_{T}\right) \lambda q_{T}}{2^{\alpha} \Pi(\lambda)-1} .
$$

If $\Pi$ is $(\operatorname{neg} l(\lambda), O(\lambda), \operatorname{poly}(\lambda))$-LRRE then $\Pi^{*}$ is $(\operatorname{neg} l(\lambda), \operatorname{poly}(\lambda), \operatorname{poly}(\lambda))$ CNMRE.

Proof. We give a reduction to the noisy-leakage resilience of $\Pi$. Before describing the reduction we introduce a sub-routine.

Procedure $\operatorname{LEAK}\left(g_{0}, g_{1}\right)$

- Let $g_{\beta, i}$ be the restriction of the function $g_{\beta}$ to the $i$-th bit.

- For $i \in[\lambda]$ send the leakage oracle query $\left(g_{0, i}, g_{1, i}\right)$ :

- let $z_{0, i}, z_{1, i}$ be the output of the oracle,

- if $z_{0, i} \neq z_{1, i}$ output $\perp$,

- if $z_{0, i}=z_{1, i}=\diamond$ output $\diamond$.

- Output $z=z_{0,0}, \ldots, z_{0, l}$.

We are now ready to describe an adversary for $\Pi^{3}$

We will keep track of the random oracle queries made by the adversary and by the tampering functions. We denote with $\mathcal{Q}_{\mathrm{A}}, \mathcal{Q}_{0}, \mathcal{Q}_{1}$ the lexicographically ordered set of tuple $x, \mathrm{RO}(x)$, and with $\overline{\mathcal{Q}}_{\mathrm{A}}, \overline{\mathcal{Q}}_{0}, \overline{\mathcal{Q}}_{1}$ the lexicographically oredered set of oracle queries (i.e., the inputs to the RO without the outputs).

Adversary $\mathrm{B}\left(\kappa_{0}, \kappa_{1}\right)$

1. Hash values $h_{0}, h_{1}$. Sample $h_{\beta} \leftarrow \$\{0,1\}^{2 \lambda}$ for $\beta \in\{0,1\}$.

2. Run the adversary $\mathrm{A}$ with input $\left(\kappa_{0}, \kappa_{1}\right)$.

3. Random oracle queries. Whenever $\mathrm{A}$ sends a query $x$ to the random oracle, forward the query to random oracle RO. Add the query $(x, \operatorname{RO}(x))$ in the set $\mathcal{Q}_{\mathrm{A}}$.

4. Tampering oracle queries. When the adversary A sends its $j$-th tampering query $\left(f_{0}^{(j)}, f_{1}^{(j)}\right)$, if the flag stop $=1$ return $\perp$, else run the sub-ruotine $\operatorname{LEAK}\left(g_{0}^{(j)}, g_{1}^{(j)}\right)$, where the leakage

3 Notice that $\Pi$ might be a randomness encoder in the standard model (i.e. no random oracle), whilst our reduction makes random oracle queries. In this case we could assume that RO is a lazy-sampled, locally-stored random function, therefore $\mathrm{B}$ would be a standard-model adversary for $\Pi$. 
functions $g_{\beta}^{(j)}$ is described below:

Leakage function $g_{\beta}^{(j)}\left(c_{\beta}\right)$ :

(a) Compile the set $\mathcal{Q}_{\beta}$ of random oracle query made by the previous tampering functions by running $f_{\beta}^{\left(j^{\prime}\right)}\left(c_{\beta}, h_{1-\beta}\right)$ for any $j^{\prime}<j$ and collecting the queries. Whenever one of the tampering functions calls $\mathrm{RO}$ on $\left(\beta \| c_{\beta}\right)$ answer with $h_{\beta}$.

(b) Compute $\left(\tilde{c}_{\beta}, \tilde{h}_{1-\beta}\right) \leftarrow f_{\beta}^{(j)}\left(c_{\beta}, h_{1-\beta}\right)$ and forward all the RO queries made by $f_{\beta}^{(j)}$ to the RO (but whenever the tampering function calls RO on $\beta \| c_{\beta}$ answer with $h_{\beta}$, instead of querying the $\mathrm{RO}$ ).

(c) If $\left(\tilde{c}_{\beta}, \tilde{h}_{1-\beta}\right)=\left(c_{\beta}, h_{1-\beta}\right)$ then output $\diamond$.

(d) If there is a tuple $\left(1-\beta \| c_{1-\beta}^{\star}, \tilde{h}_{1-\beta}\right) \in \mathcal{Q}_{\mathrm{A}} \cup \mathcal{Q}_{\beta}$,

- if $\beta=0$ then output $\operatorname{Dec}\left(\tilde{c}_{0}, c_{1}^{\star}\right)$,

- if $\beta=1$ then output $\operatorname{Dec}\left(c_{0}^{\star}, \tilde{c}_{1}\right)$,

else output $\perp$.

Let $\tilde{\mu}$ be the output of the LEAK procedure, if $\tilde{\mu}=\perp$ then set the flag stop $\leftarrow 1$. Return $\tilde{\mu}$.

5. Eventually the adversary returns a bit $b^{\prime}$. Output $b^{\prime}$.

Claim. The adversary $\mathrm{B}$ is $\left(\log \lambda+\log q_{T}+1, q_{\mathrm{RO}}\right)$-admissible.

Proof. The number of random oracle queries made by $\mathrm{B}$ is equal to the number of random oracle queries made by $A$. We can assume w.l.g. that the last tampering query of A results to self-destruct. Let $j^{*}$ be the index of the tampering query where the procedure $\operatorname{LEAK}\left(g_{\beta}^{\left(j^{*}\right)}, g_{\beta}^{\left(j^{*}\right)}\right)$ outputs $\perp$ for the first time. Let $i^{*}$ be the index of the iteration where $\operatorname{LEAK}\left(g_{\beta}^{\left(j^{*}\right)}, g_{\beta}^{\left(j^{*}\right)}\right)$ stops, we have $i^{*}<\lambda$ and let $\bar{\kappa}_{0,0}, \bar{\kappa}_{1,0}, \ldots, \bar{\kappa}_{0, i^{*}}, \bar{\kappa}_{1, i^{*}}$ be the $i^{*}$ bits leaked by the sub-routine. The adversary B leaks the values $\kappa_{\beta}^{(1)}:=g_{\beta}^{(1)}\left(c_{\beta}\right), \ldots, \kappa_{\beta}^{\left(j^{*}-1\right)}:=g_{\beta}^{\left(j^{*}-1\right)}\left(c_{\beta}\right)$ and the values $\bar{\kappa}_{\beta, 0}, \ldots, \bar{\kappa}_{\beta, i^{*}}$ to answer the tampering queries made by $\mathrm{A}$. Let $\left(x_{1}, \ldots, x_{q_{\mathrm{RO}}}\right)$ be the 
random oracle queries made by $\mathrm{B}$. For $\beta \in\{0,1\}$ :

$$
\begin{aligned}
& \left.\widetilde{\mathbb{H}}_{\infty}\left(c_{\beta} \mid h_{\beta}, c_{1-\beta}, \kappa_{\beta}^{(1)}, \ldots, \kappa_{\beta}^{\left(j^{*}-1\right)}, \bar{\kappa}_{\beta, 0}, \ldots, \bar{\kappa}_{\beta, i^{*}},\left(x_{i}, \mathrm{RO}\left(x_{i}\right)\right)_{i \in\left[q_{\mathrm{RO}}\right]}\right)\right) \\
& \quad \geq \widetilde{\mathbb{H}}_{\infty}\left(c_{\beta} \mid h_{\beta}, c_{1-\beta}, \kappa_{\beta}^{(1)}, \ldots, \kappa_{\beta}^{\left(j^{*}-1\right)}, \bar{\kappa}_{\beta, 0}, \ldots, \bar{\kappa}_{\beta, i^{*}}, \mathrm{RO}\right) \\
& \quad \geq \widetilde{\mathbb{H}}_{\infty}\left(c_{\beta} \mid h_{\beta}, c_{1-\beta}, \kappa_{1-\beta}^{(1)}, \ldots, \kappa_{1-\beta}^{\left(j^{*}-1\right)}, \bar{\kappa}_{1-\beta, 0}, \bar{\kappa}_{1-\beta, i^{*}-1}, \bar{\kappa}_{\beta, i^{*}}, \mathrm{RO}\right) \\
& \quad \geq \widetilde{\mathbb{H}}_{\infty}\left(c_{\beta} \mid h_{\beta}, c_{1-\beta}, \bar{\kappa}_{\beta, i^{*}}, i^{*}, j^{*}, \mathrm{RO}\right) \\
& \quad \geq \widetilde{\mathbb{H}}_{\infty}\left(c_{\beta} \mid h_{\beta}, c_{1-\beta}, \mathrm{RO}\right)-\left(\log \lambda+\log q_{T}+1\right) \\
& \geq \widetilde{\mathbb{H}}_{\infty}\left(c_{\beta} \mid c_{1-\beta}, \mathrm{RO}\right)-\left(\log \lambda+\log q_{T}+1\right)
\end{aligned}
$$

Where Eq. (9) follows because, by the check performed by LEAK, for any $j<j^{*}$ we have $\kappa_{0}^{(j)}=\kappa_{1}^{(j)}$ for any $i<i^{*}$ we have $\bar{\kappa}_{0, i}=\bar{\kappa}_{1, i}$, Eq. (10) follows because $\kappa_{1-\beta}^{(j)}, \bar{\kappa}_{1-\beta, i}$ are function of $c_{1-\beta}$, Eq. (11) follows by the chain rule for average conditional min-entropy and noticing that the random variable $Z_{\beta}$ and the random variable $j^{*}$ need a total of $\log q_{T}$ bits to be represented and Eq. (12) follows by independence of $h_{\beta}$.

We now analyze the advantage of B. First notice that by the claim above and taking an union bound over the elements in $\overline{\mathcal{Q}}_{\mathrm{A}} \cup \overline{\mathcal{Q}}_{1-\beta}$ we have that for $\beta \in\{0,1\}$ :

$$
\mathbb{P}\left[c_{\beta} \in \overline{\mathcal{Q}}_{\mathrm{A}} \cup \overline{\mathcal{Q}}_{1-\beta}\right] \leq\left(q_{\mathrm{RO}}+q \cdot q_{T}\right) 2^{-\alpha_{\Pi}(\lambda)+\log \lambda+\log q_{T}+1} .
$$

We condition on the event that $\forall \beta: c_{\beta} \notin \overline{\mathcal{Q}}_{\mathrm{A}} \cup \overline{\mathcal{Q}}_{1-\beta}$. Under this condition the distributions of $\left(h_{\beta}\right)_{\beta \in\{0,1\}}$ and $\left(\operatorname{RO}\left(c_{\beta}\right)\right)_{\beta \in\{0,1\}}$, given the full view of the adversary, are exactly the same, because the adversary could query $c_{\beta}$ to the random oracle only inside the tampering functions $\left(f_{\beta}^{(j)}\right)_{j \in\left[q_{T}\right]}$, but in this case the reduction would answer with $h_{\beta}$.

We further condition on the event that no collisions are found in RO on an execution of B. Notice that the probability of finding a collision is upper bounded by $\frac{\left(q_{\mathrm{RO}}+q \cdot q_{T}\right)^{2}}{2^{2 \lambda}}$.

The adversary $B$ simulates almost perfectly the experiment to $A$. Indeed, if the adversary B returns a message $\tilde{\mu} \neq \perp$ to $\mathrm{A}$ at the $j$-th tampering query then, since we assumed that there aren't collisions in the RO, it musts be that $c_{\beta}^{\star}=\tilde{c}_{\beta}$, where the former is computed by the leakage function $g_{\beta}^{(j)}$ and the latter is computed by the leakage function $g_{1-\beta}^{(j)}$.

The only difference between the simulation of $B$ and the real experiment is that, at step 4 it could happen that B returns $\perp$ but the tampering query in the real experiment would output a message different than $\perp$. 
Let $j$ be the index when this event happens for the first time. If $\mathrm{B}$ returns $\perp$ then either the procedure LEAK finds two mismatching outputs from the leakage oracle or $\exists \beta$ s.t. the leakage function $g_{\beta}^{(j)}$ outputs $\perp$.

The first case reduces to the event of finding a collision in the RO which we assumed that cannot happen, in fact, $\operatorname{Dec}\left(\tilde{c}_{0}, c_{1}^{\star}\right) \neq \operatorname{Dec}\left(c_{0}^{\star}, \tilde{c}_{1}\right)$ but $\operatorname{RO}\left(\beta \| \tilde{c}_{\beta}\right)=\operatorname{RO}\left(\beta \| c_{\beta}^{\star}\right)$ for $\beta \in\{0,1\}$. The second case instead is more interesting. In fact, $\mathcal{Q}_{A} \cup \mathcal{Q}_{\beta}$ might not cover the full set of random oracle queries that the adversary A can do through the tampering queries, thus, in principle, it could happen that the reduction cannot find a tuple $\left(1-\beta \| c_{1-\beta}^{\star}, \tilde{h}_{1-\beta}\right) \in \mathcal{Q}_{\mathrm{A}} \cup \mathcal{Q}_{\beta}$ but, nevertheless, the adversary queried $\tilde{c}_{1-\beta}=c_{1-\beta}^{\star}$ to the random oracle in one of the tampering queries $f_{1-\beta}^{\left(j^{\prime}\right)}$ for $j^{\prime} \leq j$, i.e., $\left(1-\beta \| \tilde{c}_{1-\beta}\right) \in \overline{\mathcal{Q}}_{1-\beta}$. We show that the adversary cannot guess, using the tampering query $f_{\beta}^{(j)}$, the valid value for $\tilde{h}_{1-\beta}$ that would make pass the consistency check of the decoding algorithm Dec*. Recall that we condition on $j$ being the first index where the bad event described before could happen. Thus we have that for all $j^{\prime}<j$ the output of the leakage functions $g_{0}^{\left(j^{\prime}\right)}$ and the output of $g_{1}^{\left(j^{\prime}\right)}$ agree. Also, as just said above, we condition on $\left.\left(1-\beta \| \tilde{c}_{1-\beta}\right) \in \overline{\mathcal{Q}}_{1-\beta} \wedge\left(1-\beta \| c_{1-\beta}^{\star}, \tilde{h}_{1-\beta}\right)\right) \notin \mathcal{Q}_{\beta} \cup$ $\mathcal{Q}_{\mathrm{A}}$, and we want to compute the probability that $\tilde{h}_{1-\beta}=\mathrm{RO}\left(1-\beta \| \tilde{c}_{1-\beta}\right)$.

We compute the average conditional min-entropy of $\mathrm{RO}\left(1-\beta \| \tilde{c}_{1-\beta}\right)$ given the full view of the $j$-th leakage function $g_{\beta}^{(j)}$ :

$$
\begin{array}{r}
\widetilde{\mathbb{H}}_{\infty}\left(\mathrm{RO}\left(1-\beta \| \tilde{c}_{1-\beta}\right) \mid \mathcal{Q}_{\mathrm{A}}, \mathcal{Q}_{\beta},\left(\tilde{\mu}^{\left(j^{\prime}\right)}\right)_{j^{\prime}<j}, c_{\beta}, h_{1-\beta}\right)= \\
\widetilde{\mathbb{H}}_{\infty}\left(\mathrm{RO}\left(1-\beta \| \tilde{c}_{1-\beta}\right) \mid \mathcal{Q}_{\mathrm{A}}, \mathcal{Q}_{\beta}, c_{\beta}, h_{1-\beta}\right)= \\
\widetilde{\mathbb{H}}_{\infty}\left(\mathrm{RO}\left(1-\beta \| \tilde{c}_{1-\beta}\right) \mid c_{\beta}, h_{1-\beta}\right)=2 \lambda .
\end{array}
$$

First we notice that the tuple $\left(\mathcal{Q}_{\mathrm{A}}, \mathcal{Q}_{\beta},\left(\tilde{\mu}^{\left(j^{\prime}\right)}\right)_{j^{\prime}<j}, c_{\beta}, h_{1-\beta}\right)$ is indeed the full view of the leakage function $g_{\beta}^{(j)}$, as all the randomness in the experiment comes from the random oracle queries, the challenge codeword and, possibly, the outputs of the leakage oracle. In the derivation above, the first equation holds because $\left(\tilde{\mu}^{\left(j^{\prime}\right)}\right)_{j^{\prime}<j}$ can be computed as deterministic function of $\mathcal{Q}_{\mathrm{A}}, \mathcal{Q}_{\beta}, c_{\beta}, h_{1-\beta}$, the second equation holds because we assumed that $\left(1-\beta \| c_{1-\beta}^{\star}, \tilde{h}_{1-\beta}\right) \notin \mathcal{Q}_{\mathrm{A}} \cup \mathcal{Q}_{\beta}$. This shows that the probability that $g_{\beta}^{(j)}$ computes $\tilde{h}_{1-\beta}$ at the $j$-th query equal to $\operatorname{RO}\left(1-\beta \| \tilde{c}_{1-\beta}\right)$ is $2^{-2 \lambda}$, even when $\left(1-\beta \| \tilde{c}_{1-\beta}\right) \in \overline{\mathcal{Q}}_{1-\beta}$. We can prove that the same holds when $\left(1-\beta \| \tilde{c}_{1-\beta}\right) \notin \mathcal{Q}_{1-\beta}$, in this case the value was never queried to the RO, thus the adversary can guess it with probability $2^{-2 \lambda}$. Taking an union bound over all the tampering oracle queries made by $A$ the 
probability that B outputs $\perp$ but the real experiment would have not is bounded by $q_{T} 2^{-2 \lambda}$.

Putting all together, we can conclude that the advantage of $B$ is bigger or equal to

$$
\operatorname{Adv}_{I I^{*}, \mathrm{~A}}^{\mathrm{cnmr}}(\lambda)-\frac{q_{T}}{2^{2 \lambda}}+\frac{\left(q_{\mathrm{RO}}+q \cdot q_{T}\right)^{2}}{2^{2 \lambda}}
$$

Claim. The adversary B is $\left(2 \lambda+2 \log q_{\mathrm{RO}}+\log q_{T}, q_{\mathrm{RO}}\right)$-admissible.

Proof. The number of random oracle queries made by B is equal to the number of random oracle queries made by $A$. We can assume w.l.g. that the last tampering query of A results to self-destruct. Let $j^{*}$ be the index of the tampering query where the procedure $\operatorname{LEAK}\left(g_{\beta}^{\left(j^{*}\right)}, g_{\beta}^{\left(j^{*}\right)}\right)$ outputs $\perp$ for the first time. Let $i^{*}$ be the index of the iteration where $\operatorname{LEAK}\left(g_{\beta}^{\left(j^{*}\right)}, g_{\beta}^{\left(j^{*}\right)}\right)$ stops, we have $i^{*}<\lambda$ and let $\bar{\kappa}_{0,0}, \bar{\kappa}_{1,0}, \ldots, \bar{\kappa}_{0, i^{*}}, \bar{\kappa}_{1, i^{*}}$ be the $i^{*}$ bits leaked by the sub-routine. The adversary B leaks the values $\kappa_{\beta}^{(1)}:=g_{\beta}^{(1)}\left(c_{\beta}\right), \ldots, \kappa_{\beta}^{\left(j^{*}-1\right)}:=g_{\beta}^{\left(j^{*}-1\right)}\left(c_{\beta}\right)$ and the values $\bar{\kappa}_{\beta, 0}, \ldots, \bar{\kappa}_{\beta, i^{*}}$ to answer the tampering queries made by $\mathrm{A}$. Let $\left(x_{1}, \ldots, x_{q_{\mathrm{RO}}}\right)$ be the random oracle queries made by $\mathrm{B}$. For $\beta \in\{0,1\}$ :

$$
\begin{aligned}
& \left.\widetilde{\mathbb{H}}_{\infty}\left(c_{\beta} \mid h_{\beta}, c_{1-\beta}, \kappa_{\beta}^{(1)}, \ldots, \kappa_{\beta}^{\left(j^{*}-1\right)}, \bar{\kappa}_{\beta, 0}, \ldots, \bar{\kappa}_{\beta, i^{*}},\left(x_{i}, \mathrm{RO}\left(x_{i}\right)\right)_{i \in\left[q_{\mathrm{RO}}\right]}\right)\right) \\
& \quad \geq \widetilde{\mathbb{H}}_{\infty}\left(c_{\beta} \mid h_{\beta}, c_{1-\beta}, \kappa_{\beta}^{(1)}, \ldots, \kappa_{\beta}^{\left(j^{*}-1\right)}, \bar{\kappa}_{\beta, 0}, \ldots, \bar{\kappa}_{\beta, i^{*}}, \mathrm{RO}\right) \\
& \quad \geq \widetilde{\mathbb{H}}_{\infty}\left(c_{\beta} \mid h_{\beta}, c_{1-\beta}, \kappa_{1-\beta}^{(1)}, \ldots, \kappa_{1-\beta}^{\left(j^{*}-1\right)}, \bar{\kappa}_{1-\beta, 0}, \bar{\kappa}_{1-\beta, i^{*}-1}, \bar{\kappa}_{\beta, i^{*}}, \mathrm{RO}\right) \\
& \quad \geq \widetilde{\mathbb{H}}_{\infty}\left(c_{\beta} \mid h_{\beta}, c_{1-\beta}, \bar{\kappa}_{\beta, i^{*}}, i^{*}, j^{*}, \mathrm{RO}\right) \\
& \geq \widetilde{\mathbb{H}}_{\infty}\left(c_{\beta} \mid h_{\beta}, c_{1-\beta}, \mathrm{RO}\right)-\left(\log \lambda+\log q_{T}+1\right) \\
& \geq \widetilde{\mathbb{H}}_{\infty}\left(c_{\beta} \mid c_{1-\beta}, \mathrm{RO}\right)-\left(\log \lambda+\log q_{T}+1\right)
\end{aligned}
$$

Where Eq. (9) follows because, by the check performed by LEAK, for any $j<j^{*}$ we have $\kappa_{0}^{(j)}=\kappa_{1}^{(j)}$ for any $i<i^{*}$ we have $\bar{\kappa}_{0, i}=\bar{\kappa}_{1, i}$, Eq. (10) follows because $\kappa_{1-\beta}^{(j)}, \bar{\kappa}_{1-\beta, i}$ are function of $c_{1-\beta}$, Eq. (11) follows by the chain rule for average conditional min-entropy and noticing that the random variable $Z_{\beta}$ and the random variable $j^{*}$ need a total of $\log q_{T}$ bits to be represented and Eq. (12) follows by independence of $h_{\beta}$.

Remark 2. Similarly to $[36,37,41]$, we do not consider leakage resilience for our continuous non-malleable randomness encoder. Nevertheless, our 
reduction can easily handle leakage functions by hardcoding the hash values $h_{0}, h_{1}$ and forwarding the leakage queries to its own oracle. However, there is a catch: the leakage queries sent by the adversary cannot have access to the random oracle. In fact, an attacker could forward leakage functions that make random-oracle queries on behalf of the adversary. These obfuscated random oracle queries could not be seen by our reduction thus invalidating our observability-based argument.

The Theorem 1 gives an upper bound to the advantage of any adversary against the continuous non-malleability of $\Pi^{*}$. To give a full picture, in Sec. 6 (Corollary 1) we give a lower bound based on the random-oracle query complexity and randomness complexity of the underlying randomness encoder $\Pi$. Informally, the theorem states the existence of an adversary whose random-oracle query complexity is $\Omega\left(2^{\lambda}\right)$, tampering-oracle complexity is $O(n)$ and advantage is at least $(1 / e)^{8}$.

\section{Compiler from randomness encoders to code schemes}

In this section we recall the compiler of Coretti, Faonio and Venturi [14]. The compiler makes use of an authenticated encryption scheme. Due to space constraints we defer its syntax and security definitions to Appendix ??. A $(k, m)$-SKE scheme $\Omega$ encrypts $k$-bit messages and outputs ciphertexts of size $m$. We consider the standard security property of authenticity whose security game is denoted by $\mathbf{G}_{\Omega}^{\text {auth }}$, and the standard security property of indistinghuishability which security game is denoted by $\mathbf{G}_{\Omega}^{\text {ind }}$. Let $\Pi=$ (REncode, Dec) be a $n$-randomness-encoder, and $\Omega=$ (AEnc, ADec) be a $(k, m)$-SKE scheme. Consider the following construction of a $\left(k, n^{\prime}\right)$-code $\Sigma^{\prime}=\left(\mathrm{Enc}^{\prime}, \mathrm{Dec}^{\prime}\right)$, where $n^{\prime}:=m+n$.

$\operatorname{Enc}^{\prime}(s)$ : Upon input a value $s \in\{0,1\}^{k}$, compute $c_{0}, c_{1} \leftarrow \$ \operatorname{REncode}\left(1^{\lambda}\right)$, let $\kappa \leftarrow \operatorname{Dec}\left(c_{0}, c_{1}\right)$, and compute $\gamma \leftarrow \$ \operatorname{AEnc}(\kappa, s)$; return $c_{0}^{\prime}, c_{1}^{\prime}$ where $c_{\beta}^{\prime}=\left(c_{\beta}, \gamma\right)$ for $\beta \in\{0,1\}$.

$\operatorname{Dec}^{\prime}\left(c_{0}^{\prime}, c_{1}^{\prime}\right)$ : Parse $c_{\beta}^{\prime}:=\left(c_{\beta}, \gamma_{\beta}\right)$ for $\beta \in\{0,1\}$. If $\gamma_{0} \neq \gamma_{1}$, return $\perp$ and self destruct; else let $\tilde{\kappa}=\operatorname{Dec}\left(c_{0}, c_{1}\right)$. If $\tilde{\kappa}=\perp$, return $\perp$ and self destruct; else return the same as $\operatorname{ADec}\left(\tilde{\kappa}, \gamma_{0}\right)$.

The difference between the compiler $\Sigma$ described above and the compiler of [14] is that our compiler starts from a CNMRE, while their construction starts from a noisy-leakage-resilient CNMC. In particular, their proof strategy relies on the noisy-leakage resilience of the underlying CNMC 
while our proof strategy does not. A similar strategy to ours was recently used by Brian, Faonio and Venturi in the context of continuous non-malleable secret sharing schemes [10].

Theorem 2. For any adversary A which makes at most $q_{T}:=q_{T}(\lambda)$ tampering oracle queries there exist adversaries $\mathrm{B}$ which makes at most $(m+1) \cdot q_{T}$ tampering oracle queries, and adversaries $\mathrm{B}^{\prime}$ and $\mathrm{B}^{\prime \prime}$ such that

$$
\mathbf{A d v}_{\Sigma, \mathbf{A}}^{\mathrm{cnmc}}(\lambda) \leq 2 \mathbf{A} \mathbf{d} \mathbf{v}_{I, \mathrm{~B}}^{\mathrm{cnmr}}(\lambda)+q_{T} \cdot \mathbf{A d v}_{\Omega, \mathrm{B}^{\prime}}^{\text {auth }}(\lambda)+\mathbf{A} \mathbf{d} \mathbf{v}_{\Omega, \mathrm{B}^{\prime \prime}}^{\text {ind }}(\lambda) .
$$

Proof. The proof proceeds by a sequence of hybrid experiments. We assume, w.l.o.g., that $\mathbb{P}\left[\mathbf{G}_{\Sigma, A}^{\mathrm{cnm}}\left(1^{\lambda}, \mu_{0}, \mu_{1}\right)=1\right] \geq \frac{1}{2}$. We $\underline{\text { underline in gray }}$ the differences between consecutive hybrids. Fix $\mu_{0}, \mu_{1}$ and let $\mathbf{H}_{1}\left(1^{\lambda}\right)$ be the same as $\mathbf{G}_{\Pi, \mathbf{A}}^{\mathrm{cnm}}\left(1^{\lambda}, \mu_{0}, \mu_{1}\right)$ but where:

- The target codeword is generated by computing $c_{0}, c_{1} \leftarrow \$ \operatorname{REncode}\left(1^{\lambda}\right)$, setting $\kappa_{1} \leftarrow \operatorname{Dec}\left(c_{0}, c_{1}\right)$, sampling $\kappa_{0} \leftarrow \$\{0,1\}^{\lambda}$ and computing the chipertext $\gamma \leftarrow \$ \operatorname{AEnc}\left(\kappa_{0}, s\right)$;

- At each tampering query, let $f=\left(f_{0}, f_{1}\right)$ be the tampering function sent by the adversary and compute, let $\left(\tilde{c}_{\beta}, \tilde{\gamma}_{\beta}\right)=f_{\beta}\left(c_{\beta}^{\prime}\right)$ for $\beta \in$ $\{0,1\}$. The decoding algorithm checks if $\operatorname{Dec}\left(\tilde{c}_{0}, \tilde{c}_{1}\right)=\kappa_{1}$, if this is the case (and if $\tilde{\gamma}_{0}=\tilde{\gamma}_{1}$ ) then it decrypts the tampered ciphertext $\tilde{\gamma}_{0}$ using the key $\kappa_{0}$.

Consider the following reduction:

Adversary $\mathrm{B}\left(1^{\lambda}, \kappa_{0}, \kappa_{1}\right)$ :

1. Sample a random bit $b^{*}$ and set $\gamma \leftarrow \operatorname{AEnc}\left(\kappa_{0}, \mu_{b^{*}}\right)$. Compute an auxiliary codewords $c_{0}^{(\alpha)}, c_{1}^{(\alpha)} \leftarrow \operatorname{REncode}\left(1^{\lambda}\right)$ such that $\operatorname{Dec}\left(c_{0}^{(0)}, c_{1}^{(1)}\right)=\operatorname{Dec}\left(c_{0}^{(1)}, c_{1}^{(0)}\right)=\perp$ and let $\kappa^{(\alpha)} \leftarrow \operatorname{Dec}\left(c_{0}^{(\alpha)}, c_{1}^{(\alpha)}\right)$ for $\alpha \in\{0,1\}$. Start the adversary $\mathrm{A}\left(1^{\lambda}, \mu_{0}, \mu_{1}\right)$, set the flag stop $\leftarrow 0$.

2. Random oracle queries. Whenever $\mathrm{A}$ sends a query $x$ to the random oracle, forward the query to random oracle RO.

3. Tampering oracle queries. When the adversary A sends its $j$-th tampering query $\left(f_{0}^{(j)}, f_{1}^{(j)}\right)$, if the flag stop $=1$ return $\perp$ else consider the following tampering function:

Tampering function $f_{\beta}^{j, i}\left(c_{\beta}\right)$ :

(a) Compute $\left(\tilde{c}_{\beta}, \tilde{\gamma}_{\beta}\right) \leftarrow f_{\beta}^{(j)}\left(c_{\beta}, \gamma\right)$; 
(b) Let $\alpha$ be the $i$-th bit of $\tilde{\gamma}_{\beta}$, return $c_{\beta}^{(\alpha)}$.

For $i \in[m]$ send the tampering query $\left(f_{0}^{j, i}, f_{1}^{j, i}\right)$ and:

- If the tampering oracle outputs $\kappa^{(\alpha)}$ then set $\alpha_{i} \leftarrow \alpha$;

- Else the tampering oracle outputs $\perp$ then return $\perp$ to A.

Let $\tilde{\gamma}=\left(\alpha_{0}, \ldots, \alpha_{m-1}\right)$. Consider the tampering function $f_{\beta}^{\prime}\left(c_{\beta}\right)$

that computes $\left(\tilde{c}_{\beta}, \tilde{\gamma}\right) \leftarrow f_{\beta}^{(j)}\left(c_{\beta}, \gamma\right)$ and outputs $\tilde{c}_{\beta}$.

Send the tampering query $\left(f_{0}^{\prime}, f_{1}^{\prime}\right)$ and obtain $\tilde{\kappa}$.

- If $\tilde{\kappa}=\perp$ return $\perp$ to $A$ and set the flag stop $\leftarrow 1$,

- if $\tilde{\kappa}=\diamond$ then set $\tilde{\kappa} \leftarrow \kappa_{0}$,

- compute $\tilde{\mu} \leftarrow \operatorname{ADec}(\tilde{\kappa}, \tilde{\gamma})$ if $\tilde{\mu}=\perp$ then set the flag stop $\leftarrow$ 1 and return $\perp$ to $\mathrm{A}$ else return $\tilde{\mu}$.

4. Eventually the adversary return a bit $b^{\prime}$, return $b^{*}=b^{\prime}$.

Claim. $\mathbb{P}\left[\mathbf{G}_{\Sigma, \mathbf{A}}^{\mathrm{cnmc}}\left(1^{\lambda}, \mu_{0}, \mu_{1}\right)=1\right] \leq 2 \mathbf{A d v}_{I, \mathrm{~B}}^{\mathrm{cnmm}}\left(1^{\lambda}\right)+\mathbb{P}\left[\mathbf{H}_{1}\left(1^{\lambda}\right)=1\right]$.

Proof (of the Claim). It is sufficient to prove that $\mathbb{P}\left[\mathbf{G}_{\Pi, \mathrm{B}}^{\mathrm{cnm}}\left(1^{\lambda}, \mu_{0}, \mu_{1}\right)=1 \mid b=0\right]=$ $\mathbb{P}\left[\mathbf{G}_{\Sigma, \mathrm{A}}^{\mathrm{cnm}}\left(1^{\lambda}\right)=1\right], \mathbb{P}\left[\mathbf{G}_{\Pi, \mathrm{B}}^{\mathrm{cnmm}}\left(1^{\lambda}, \mu_{0}, \mu_{1}\right)=0 \mid b=1\right]=\mathbb{P}\left[\mathbf{H}_{1}\left(1^{\lambda}\right)=1\right]$. First we notice that at step 1 of $\mathrm{B}$, the reduction samples two auxiliary codewords $c^{(0)}$ and $c^{(1)}$ such that $\operatorname{Dec}\left(c_{0}^{(0)}, c_{1}^{(1)}\right)=\operatorname{Dec}\left(c_{0}^{(1)}, c_{1}^{(0)}\right)=\perp$. If such constraint does not hold then we can break the non-malleability of $\Sigma$ by applying Thm. 5. Also notice that, assuming that the constraint does not hold, then the advantage of the reduction to non-malleability of $\Sigma$ is tighter than the advantage of $B$. Thus we assume that the condition holds. Independently of the challenge bit $b$, the adversary $\mathrm{B}$ simulates perfectly the tampering oracle queries. In fact, if the $j$-th tampering oracle query of $\mathrm{A}$, on input the tampering function $\left(f_{0}^{(j)}, f_{1}^{(j)}\right)$, outputs $\perp$ then either (1) the ciphertexts $\tilde{\gamma}_{0}$ and $\tilde{\gamma}_{1}$ are different or $(2) \operatorname{Dec}\left(\tilde{c}_{0}, \tilde{c}_{1}\right)=\perp$ or $(3)$ $\operatorname{ADec}\left(\tilde{\kappa}, \tilde{\gamma}_{0}\right)=\perp$. If the event (1) happens, let $i$ be the first index where the ciphertexts $\tilde{\gamma}_{0}$ and $\tilde{\gamma}_{1}$ differ, the tampering query $\left(f_{0}^{j, i}, f_{1}^{j, i}\right)$ returns either $c_{0}^{(0)}, c_{1}^{(1)}$ or $c_{0}^{(1)}, c_{1}^{(0)}$ which makes $\mathrm{B}$ to return $\perp$ to $\mathrm{A}$. In the event (2) happens, then $B$ would receive $\perp$ from the tampering oracle query with input $\left(f_{0}^{\prime}, f_{1}^{\prime}\right)$. If the event (3) happens, then by definition of $\mathrm{B}$, it would return $\perp$ to $A$.

On the other hand, if the $j$-th tampering oracle query of $A$ outputs a message $\tilde{\mu} \neq \perp$ then, by inspection of $B$, it is easy to show that $B$ would return the same message $\tilde{\mu}$ to $\mathrm{A}$.

Finally notice that if $b=0$ then the target codeword for $\Pi$ encodes $\kappa_{0}$, thus the codeword $\left(c_{0}, \gamma\right),\left(c_{1}, \gamma\right)$ is distributed exactly as the output of 
Enc ${ }^{\prime}$. Else, if $b=1$ the target codeword for $\Pi$ encodes $\kappa_{1}$ so the codeword is distributed exactly as generated by $\mathbf{H}_{1}$.

Let $\mathbf{H}_{2}$ be the same as $\mathbf{H}_{1}$ but where for any tampering query $\left(f_{0}, f_{1}\right)$ let $\left(\tilde{c}_{0}, \tilde{\gamma}\right),\left(\tilde{c}_{1}, \tilde{\gamma}^{\prime}\right)$ be the tampered codeword as computed by the tampering oracle, if $\operatorname{Dec}\left(\tilde{c}_{0}, \tilde{c}_{1}\right)=\kappa_{1}$ and $\tilde{\gamma} \neq \gamma$ then the hybrid $\mathbf{H}_{2}$ directly returns $\perp$ and self destructs else, if $\operatorname{Dec}\left(\tilde{c}_{0}, \tilde{c}_{1}\right)=\kappa_{1}$ and $\tilde{\gamma}=\gamma$, it directly returns $\diamond$. It is easy to show that, by reduction to the authenticity of $\Omega$, the advantages of $\mathbf{H}_{2}$ and $\mathbf{H}_{3}$ are negligibly close.

Let $\mathbf{H}_{3}$ be the same as $\mathbf{H}_{2}$ but where the ciphertext in the target codeword is computed as $\gamma \leftarrow \operatorname{AEnc}\left(\kappa_{0}, 0^{k}\right)$. We can show that, by reduction to the indistinguishability of $\Omega$, the advantages of $\mathbf{H}_{2}$ and $\mathbf{H}_{3}$ are negligibly close. Finally, it is easy to show that the advantage in $\mathbf{H}_{3}$ is $\frac{1}{2}$.

\section{Our Leakage-Resilient Randomness Encoders}

We give two constructions $\Pi_{1}$ and $\Pi_{2}$ of LRREs, due to space constraints the proofs of security of $\Pi_{1}$ and $\Pi_{2}$ appear in the full version [25]. Notably the randomness encoder $\Pi_{2}$ has optimal leakage parameter, namely the leakage parameter is only $\lambda$ bits smaller than the size of the codeword.

Let $p$ be a prime such that $p \geq 2^{\lambda}$ and let $m \in \mathbb{N}$. Consider the following $(m \log p)$-randomness-encoder $\Pi_{1}=\left(\right.$ REncode $_{1}$, Dec $\left._{1}\right)$ :

REncode $_{1}\left(1^{\lambda}\right)$ : Sample column vectors $\boldsymbol{x}_{0}, \boldsymbol{x}_{1} \leftarrow \$ \mathbb{Z}_{p}^{m}$. Output $c_{0}, c_{1}$ where $c_{\beta}$ is the binary representation of $x_{\beta}$

$\operatorname{Dec}_{1}\left(c_{0}, c_{1}\right)$ : Parse $c_{\beta}$ as a vector $\boldsymbol{x}_{\beta} \in \mathbb{Z}_{p}^{m}$. Return the binary representation of $\boldsymbol{x}_{0}^{T} \cdot \boldsymbol{x}_{1} \in \mathbb{Z}_{p}$.

Theorem 3. Let $\ell \leq m \log p$, for any $q$, the $\Pi_{1}$ scheme is $\left(O\left(2^{-\lambda}\right), \ell, q\right)$ noisy-leakage resilient for $\ell \leq(m+1) \log p / 2-2 \lambda$. In more detail, for any $(\ell, q)$-admissible adversary $\mathrm{A}: \operatorname{Adv}_{\Pi_{1}, \mathrm{~A}}^{\operatorname{Irs}}(\lambda) \leq 2^{-(m-1) \log p / 2+\ell}$.

The theorem follows easily from the following two lemmas. The first lemma proves that the inner product over large field is an average case two-source extractor. the lemma is taken from Dodis et al. [19] (Lemma B.2 in full version). The second lemma was proved by Dziembowski and Pietrzak [23].

Lemma 2. Let $\mathbf{u}$ be uniformly random over $\mathbb{Z}_{p}$, for any unbounded distinguisher $\mathrm{D}$, any random variables $\boldsymbol{x}_{0}, \boldsymbol{x}_{1} \in \mathbb{Z}_{p}^{m}, \mathbf{Z} \in\{0,1\}^{*}$ such that $\boldsymbol{x}_{0}$ 
and $\boldsymbol{x}_{1}$ are independent conditioned on $\mathbf{Z}$ :

$$
\left|\mathbb{P}\left[\mathrm{D}\left(\boldsymbol{x}_{0}^{T} \cdot \boldsymbol{x}_{1}, \mathbf{Z}\right)=1\right]-\mathbb{P}[\mathrm{D}(\mathbf{u}, \mathbf{Z})=1]\right| \leq 2^{\left((m+1) \log p-\ell_{0}-\ell_{1}\right) / 2} .
$$

where $\ell_{\beta}=\widetilde{\mathbb{H}}_{\infty}\left(\boldsymbol{x}_{\beta} \mid \mathbf{Z}\right)$.

The second lemma proves that independence is maintained even after split-state leakage.

Lemma 3. Let $\boldsymbol{x}_{0}$ and $\boldsymbol{x}_{1}$ be two independent random variables. For any adversary $\mathbf{z} \leftarrow \mathrm{A}^{\mathcal{O}_{\text {leak }}\left(\left(\boldsymbol{x}_{0}, \boldsymbol{x}_{1}\right), \cdot, \cdot\right)}$, the random variables $\left(\boldsymbol{x}_{1} \mid \mathbf{z}\right)$ and $\left(\boldsymbol{x}_{0} \mid \mathbf{z}\right)$ are independent.

Proof (of Thm. 3). First notice that the randomness encoder $\Pi_{1}$ does not use the random oracle, so queries to the random oracle have no impact in our security analysis. Recall that in the game $\mathbf{G}_{\Pi_{1}, \mathrm{~A}}^{\text {Irre }}$ the adversary receives two keys $\kappa_{0}$ and $\kappa_{1}$, it has leakage oracle access to the codeword and it outputs a bit. Consider the hybrid experiment $\mathbf{H}$ that is identical to $\mathbf{G}^{\text {Irre }}$ but where both the key $\kappa_{0}$ and $\kappa_{1}$ are chosen uniformly at random from $\{0,1\}^{\lambda}$. It is not hard to see that $\mathbb{P}[\mathbf{H}=1]=\frac{1}{2}$. We show that the distribution $\mathbf{G}_{\Pi_{1}, \mathrm{~A}}^{\text {Ire }}$ and the distribution $\mathbf{H}$ are statistically close.

Let $\mathbf{Z}$ be the state of $\mathbf{A}$ before it outputs its guess. W.l.o.g. the state contains all the leakage oracle queries and answers and all the randomness used by $A$. By Lemma 3 we have $\left(\boldsymbol{x}_{0} \mid \mathbf{Z}\right)$ and $\left(\boldsymbol{x}_{1} \mid \mathbf{Z}\right)$ are independent, moreover for $\beta \in\{0,1\}: \widetilde{\mathbb{H}}_{\infty}\left(\boldsymbol{x}_{\beta} \mid \mathbf{Z}\right) \geq m \log p-\ell$, thus by Lemma 2 the theorem follows.

Let $(m-1) \log p / 2 \geq n$ and let $\mathrm{RO}$ be a random oracle ${ }^{4}$ with output $\mathbb{Z}_{p}^{m}$. Consider the following $n$-randomness-encoder $\Pi_{2}=\left(\right.$ REncode $_{2}$, Dec $\left._{2}\right)$.

REncode $\left(1^{\lambda}\right)$ : Sample and output $c_{0}, c_{1} \leftarrow \$\{0,1\}^{n}$.

$\operatorname{Dec}\left(c_{0}, c_{1}\right)$ : Compute $\boldsymbol{x}_{i} \leftarrow \mathrm{RO}\left(c_{i}\right)$ for $i \in\{0,1\}$ and return the binary representation of $\boldsymbol{x}_{0}^{T} \cdot \boldsymbol{x}_{1}$.

Theorem 4. Let $\ell+\lambda \leq n$, for any $q_{\mathrm{RO}}(\lambda) \in \mathbb{N}$, the encoding scheme $\Pi_{2}$ is $\left(O\left(2^{-\lambda} q_{\mathrm{RO}}\right), \ell, q_{\mathrm{RO}}\right)$-noisy-leakage resilient. In more detail, for any $\left(\ell, q_{\mathrm{RO}}\right)$-admissible adversary A: $\operatorname{Adv}_{\Pi_{2}, \mathrm{~A}}^{\mathrm{Irs}}(\lambda) \leq 2^{\ell-n}\left(2 q_{\mathrm{RO}}+2\right)$.

The idea for the proof is that the adversary can either leak from $c_{i}$ or directly from $\mathrm{RO}\left(c_{i}\right)$. The former kind of leakage cannot give any advantage to the adversary, since the adversary should be able to guess $n-\ell \geq \lambda$ bits to obtain any information about $\mathrm{RO}\left(c_{i}\right)$, the latter form of leakage is protected by the same argument of the leakage resilience of $\Pi_{1}$.

\footnotetext{
${ }^{4}$ It can be easily realized using a $\mathrm{RO}^{\prime}$ with codomain $\{0,1\}^{2 \lambda}$.
} 
Proof. We reduce to the security of $\Pi_{1}$. Consider the hybrid experiment $\mathbf{H}_{\mathrm{A}}$ equivalent to the $\mathbf{G}_{\Pi_{2}, \mathrm{~A}}^{\text {Irre }}$ experiment but where the output of the experiment is a random bit if $\mathrm{A}$ queries the random oracle either on $c_{0}$ or on $c_{1}$.

Claim. $\left|\mathbb{P}\left[\mathbf{G}_{\Pi_{2}, \mathrm{~A}}^{\text {Ire }}(\lambda)=1\right]-\mathbb{P}\left[\mathbf{H}_{\mathrm{A}}(\lambda)=1\right]\right| \leq 2 q_{\mathrm{RO}} 2^{\ell-n}$

Proof. The two experiments proceed exactly the same until the bad event that the adversary A queries the random oracle on $c_{0}$ or on $c_{1}$ happens. Let us call such event Bad. Therefore we need only to bound the probability of Bad. Let $\operatorname{Bad}_{i, b}$ the event that A queries $c_{b}$ at its $i$-th query to RO.

$$
\mathbb{P}[\mathrm{Bad}] \leq \sum_{i \in\left[q_{\mathrm{RO}}\right], b \in\{0,1\}} \mathbb{P}\left[\operatorname{Bad}_{i, b}\right] \leq 2 q_{\mathrm{RO}} \max _{i, b} \mathbb{P}\left[\operatorname{Bad}_{i, b}\right]
$$

Let $\mathbf{z}_{i}$ be the state of the adversary at the $i$-th query (which includes also all the random-oracle queries made), by the definition of average conditional min-entropy we have that $\mathbb{P}\left[\operatorname{Bad}_{i, b}\right] \leq 2^{-\widetilde{\mathbb{H}}_{\infty}\left(c_{b} \mid \mathbf{z}_{i}\right)}$. In fact, we can define a predictor that runs $A$ with state set to $\mathbf{z}_{i}$ and outputs as its own guess the random oracle query made by $\mathrm{A}$. By the $\left(\ell, q_{\mathrm{RO}}\right)$ admissibility of $\mathrm{A}$ we have that $\widetilde{\mathbb{H}}_{\infty}\left(c_{b} \mid \mathbf{z}_{i}\right) \geq \widetilde{\mathbb{H}}_{\infty}\left(c_{b} \mid c_{1-b}, \mathrm{RO}\right)-\ell=n-\ell$.

Claim. For any $\left(\ell, q_{\mathrm{RO}}\right)$-admissible $\mathrm{A}$ there exists an $(\ell, 0)$-admissible $\mathrm{A}^{\prime}$ such that: $\left|\mathbb{P}\left[\mathbf{H}_{\mathbf{A}}(\lambda)=1\right]-1\right| / 2=\mathbf{A d v}_{\Pi_{1}, \mathbf{A}^{\prime}}^{\text {Irs }}(\lambda)$.

Proof. Let $\mathrm{A}^{\prime}$ be the adversary that simulates $\mathrm{A}$ and keeps the list $\mathcal{Q}$ of random oracle queries made by $A$. Additionally, $A^{\prime}$ samples a random function $\mathcal{H}$ with domain $\{0,1\}^{n}$ and co-domain $\mathbb{Z}_{p}^{m}$ and answers the random oracle query of $A$ using $\mathcal{H}$. (Notice that $A^{\prime}$ does not need to be efficiently computable.) Also, $\mathrm{A}^{\prime}$ samples two random values $c_{0}, c_{1} \leftarrow \$\{0,1\}^{n}$. Whenever the adversary $\mathrm{A}$ sends a leakage oracle query $\left(g_{0}, g_{1}\right)$ the adversary $\mathrm{A}^{\prime}$ sends the leakage oracle query $\left(g_{0}^{\prime}, g_{1}^{\prime}\right)$ where the leakage functions $g_{\beta}^{\prime}$ for $\beta \in\{0,1\}$ are defined below:

Leakage function $g_{\beta}^{\prime}(x)$ :

$-\operatorname{Run} g_{\beta}\left(c_{\beta}\right)$

- Upon random oracle query $z$ from $g_{\beta}$ if $z=c_{\beta}$ then return $x$ else return $\mathcal{H}(z)$ to $g_{\beta}$.

- Output what $g_{\beta}$ does.

Eventually A outputs its guess $b$, the adversary $\mathrm{A}^{\prime}$ first checks that $c_{\beta} \notin \mathcal{Q}$ for $\beta \in\{0,1\}$, if so it returns a random bit else $A^{\prime}$ returns $b$. 
Notice that $A^{\prime}$ simulates perfectly the hybrid experiment as long as neither $c_{0}$ nor $c_{1}$ are queried to the random oracle by $A$. In fact, if we condition on $\forall \beta: c_{\beta} \notin \mathcal{Q}$ then the adversary $\mathrm{A}^{\prime}$ is running the hybrid experiment $\mathbf{H}$ with the random oracle $\mathcal{H}^{\prime}(x)$ that answers $\mathcal{H}(x)$ if $x \notin$ $\left\{c_{0}, c_{1}\right\}$, with $\boldsymbol{x}_{0}$ if $x=c_{0}$ and with $\boldsymbol{x}_{1}$ if $x=c_{1}$, where $\left(\boldsymbol{x}_{0}, \boldsymbol{x}_{1}\right)$ is the challenge codeword for $A^{\prime}$. On the other hand, when this bad event happens the adversary $A^{\prime}$ will surely outputs a random bit, as the hybrid experiment does.

Putting the claims together, by the triangular inequality, and because of the relation $(m-1) \log p / 2+\lambda \geq n$ we have the statement of the theorem.

\subsection{Instantiations}

We present two instantiations for our continuous non-malleable randomness encoders. By joining together the results of Thm. 1 and Thm. 3 we obtain a $(m \log p+2 \lambda)$-randomness-encoders scheme $\Pi_{1}^{*}$ with concrete security being:

$$
\begin{aligned}
\max _{\mathrm{A}} \mathbf{A d v}_{\Pi_{1}^{*}, \mathrm{~A}}^{\mathrm{cnmre}}(\lambda) \leq & \exp \left(-(m-1) \log p / 2+\log \lambda+\log q_{T}+1\right) \\
& +\frac{q_{T}+\left(q_{\mathrm{RO}}+q \cdot q_{T}\right)^{2}}{2^{2 \lambda}}+\frac{\left(q_{\mathrm{RO}}+q \cdot q_{T}\right) \lambda q_{T}}{2^{m \log p-1}} .
\end{aligned}
$$

For concreteness, suppose that an adversary can make $q_{\mathrm{RO}}+q \cdot q_{T}=2^{40}$ random-oracle queries and $q_{T}=2^{20}$ tampering-oracle queries, then to have $\approx 128$-bits of security we need to set $(m-1) \log p \geq 312$ and $p \geq 2^{128}$, for example we can set we $m=2$ and $p \geq 2^{312}$. Instantiating the random oracle using SHA256 then the codeword size would be approximately $2 \times 880$ bits. The time complexity of the decoding algorithm would be approximately the same as two SHA256 functions plus an inner-product between two vectors in $\mathbb{Z}_{p}^{m}$. Our second instantiation is derived by joining together the results of Thm. 1 and Thm. 4 . We obtain a $n+\lambda$-randomnessencoders scheme $\Pi_{2}^{*}$ with concrete security being:

$$
\begin{aligned}
\max _{\mathrm{A}} \mathbf{A d v}_{\Pi_{2}^{*}, \mathrm{~A}}^{\mathrm{cnmre}}(\lambda) \leq & \exp \left(-n+\log \lambda+\log q_{T}+\log q_{\mathrm{RO}}+2\right) \\
& +\frac{q_{T}+\left(q_{\mathrm{RO}}+q \cdot q_{T}\right)^{2}}{2^{2 \lambda}}+\frac{\left(q_{\mathrm{RO}}+q \cdot q_{T}\right) \lambda q_{T}}{2^{m \log p-1}} .
\end{aligned}
$$

Assuming the same setup of before, to get $\approx 128$-bits of security we need to set $n \geq 128+69$. Using SHA256 the codeword size would be approximately $2 \times 453$ bits. The time complexity of the decoding algorithm would be the same of 8 SHA256 functions. In particular, the size of the codeword is in total only $\approx 7$ times bigger than the size of the derived key. 


\section{Lower Bounds for CNMREs in the ROM}

Definition 8. Given a n-randomness encoder $\Pi$, an algorithm $\mathrm{A}$ is a $\left(\epsilon, q_{\mathrm{RO}}\right)$-finder for $\Pi$ if $\mathrm{A}\left(1^{\lambda}\right)$ makes at most $q_{\mathrm{RO}}(\lambda)$ random oracle queries and if:

$\mathbb{P}\left[\begin{array}{c}\perp \neq \operatorname{Dec}\left(c_{0}, c_{1}\right) \neq \operatorname{Dec}\left(c_{0}, c_{1}^{\prime}\right) \neq \perp:\left(c_{0}, c_{1}, c_{0}^{\prime}, c_{1}^{\prime}\right) \leftarrow \mathrm{A}^{\mathrm{RO}}\left(1^{\lambda}\right) \\ \left(c_{0}=c_{0}^{\prime}\right) \vee\left(c_{1}=c_{1}^{\prime}\right)\end{array}\right] \geq \epsilon(\lambda)$

In the next theorem first we show that the existence of a finder is sufficient to break continuous non-malleability, then we show that even if there is no finder, we still can break continuous non-malleability given enough random oracle queries.

Theorem 5. Let $n(\lambda) \in \mathbb{N}$ and let $\Pi$ be a n-randomness encoder:

1. If there exists a $\left(\epsilon, q_{\mathrm{RO}}\right)$-finder for $\Pi$ then for any $\frac{\epsilon}{2} \geq \delta>0$, $\Pi$ is not a $\left(\frac{\epsilon}{2}-\delta, 0, q_{\mathrm{RO}}\right)-C N M R E$. Namely, there exists an adversary $\mathrm{A}^{\prime}$ making up to $q_{\mathrm{RO}}$ random oracle queries and $n+1$ tampering queries from $\mathcal{F}_{n, 0}$, such that $\frac{\epsilon}{2} \leq \mathbf{A d v}_{\Pi, \mathbf{A}^{\prime}}^{\text {cnmre }}\left(1^{\lambda}\right)$.

2. Suppose that $\operatorname{Enc}\left(1^{\lambda}\right)$ makes at most $q_{\mathrm{RO}}^{\mathrm{Enc}}(\lambda)$ random oracle queries and uses $r(\lambda)$ random bits. If for any $\epsilon, q_{\mathrm{RO}}^{\prime}$, there does not exist a $\left(\epsilon, q_{\mathrm{RO}}^{\prime}\right)$-finder then for any $\delta>0$ any $q_{\mathrm{RO}}, q$ such that $q_{\mathrm{RO}}+q \geq$ $2^{r} \cdot q_{\mathrm{RO}}^{\mathrm{Enc}}$ the scheme $\Pi$ is not a $\left(1 / 2-\delta, q, q_{\mathrm{RO}}\right)-C N M R E$. Namely, there exists an adversary $\mathrm{A}^{\prime}$ making up to $q_{\mathrm{RO}}$ random oracle queries and 1 tampering query from $\mathcal{F}_{n, q}$ such that $\mathbf{A d v}_{\Pi, \mathbf{A}^{\prime}}^{\mathrm{cnmme}}\left(1^{\lambda}\right)=1 / 2$.

Proof (Sketch). For the first part of the theorem let the adversary $\mathrm{A}^{\prime}$ first run the finder algorithm. For simplicity, let us assume that the finder outputs a tuple $\left(c_{0}, c_{1}, c_{0}^{\prime}, c_{1}^{\prime}\right)$ where $c_{0}=c_{0}^{\prime}$. If the output of the finder is not valid then the adversary $\mathrm{A}^{\prime}$ outputs a random bit. Else, for $i=$ $0, \ldots, n-1$ sends the tampering query $\left(f_{0}^{(i)}, f_{1}^{(i)}\right)$ where $f_{0}^{(i)}$ returns $c_{0}$ and $f_{1}^{(i)}\left(c_{1}^{*}\right)$ returns either $c_{1}$ or to $c_{1}^{\prime}$ depending on the on the $i$-th bit of $c_{1}^{*}$. After this process, the adversary can extract in full the value $c_{1}^{*}$ of the target codeword, thus it can send a last tampering query that breaks non-malleability. It is clear that the adversary wins the game $\mathbf{G}^{\text {cnmre }}$ with probability at least $\epsilon+(1-\epsilon) \frac{1}{2}$.

For the second part of the theorem, for simplicity we consider first the case where $q=0$. Since no finder exists, then for any $c_{0}$ there exists unique $c_{1}$ such that $\left(c_{0}, c_{1}\right) \in\left\{\operatorname{Enc}\left(1^{\lambda} ; \rho\right): \rho \in\{0,1\}^{r}\right\}$. Thus the the adversary 
$\mathrm{A}^{\prime}$ can compile a bijection $L_{0}$ such that $L_{0}\left(c_{0}\right)=c_{1}$, also let $L_{1}$ be the inverse of $L_{0}$. To compile such bijections the adversary $\mathrm{A}^{\prime}$ needs at most $2^{r} \cdot q_{\mathrm{RO}}^{\mathrm{Enc}}$ random oracle queries. Then given such bijections, the adversary sends the tampering queries $f_{0}, f_{1}$ where $f_{\beta}\left(c_{\beta}\right)$ computes $c_{1-\beta} \leftarrow L_{\beta}\left(c_{\beta}\right)$, decodes $\kappa \leftarrow \operatorname{Dec}\left(c_{0}, c_{1}\right)$, and if $\kappa=\kappa_{0}$ sets the codeword to $\perp$, else leaves the codeword untouched. It is clear that the adversary wins the game $\mathbf{G}^{\text {cnmre }}$ with probability 1 .

For the case $q>0$, we can consider an adversary that computes first partially the bijection $L_{0}$ using the budget of random-oracle queries $q_{\mathrm{RO}}$ and then finishes to computes the bijection $L_{0}$ using the budget of random-oracle queries that the tampering function can use.

Corollary 1. Let $\Pi=$ (REncode, Dec) be a randomness-encoder where REncode $\left(1^{\lambda}\right)$ makes up to $q_{\mathrm{RO}}^{\mathrm{REncode}}(\lambda)$ queries to the random oracle and uses at most $r(\lambda)$ bits of randomness, and Dec makes up to $q_{\mathrm{RO}}^{\mathrm{Dec}}(\lambda)$ queries to the random oracle. Also, let $\Pi$ be $\left(\epsilon, \ell, 2^{r}\left(q_{\mathrm{RO}}^{\mathrm{RE} n c o d e}+q_{\mathrm{RO}}^{\mathrm{Dec}}\right)\right)$-noisy leakage resilient in the $R O M$, for any $\epsilon, \ell$ where $\ell \geq 2$.

Consider $\Pi^{*}$ be our CNMRE from Sec. 3 instantiated with the randomness encoder $\Pi$. There exists an adversary $\mathrm{A}$ that makes up to $2^{r}\left(q_{\mathrm{RO}}^{\mathrm{RE} c o d e}+\right.$ $\left.q_{\mathrm{RO}}^{\mathrm{Dec}}\right)+2^{\lambda}$ random oracle queries and up to $n+1$ tampering oracle queries from $\mathcal{F}_{n+2 \lambda, 0}$ such that:

$$
(1 / e)^{\left(\left(\frac{1}{2}-\epsilon\right) 2^{\lambda}-1\right)^{2} / 2^{2 \lambda-1}} \leq \mathbf{A d v}_{\Pi{ }^{*}, \mathbf{A}}^{\mathrm{cnmre}}(\lambda) .
$$

In particular, when $\epsilon(\lambda) \in \operatorname{negl}(\lambda)$ then $(1 / e)^{8} \leq \mathbf{A d v}_{\Pi^{*}, A}^{\mathrm{cnmre}}(\lambda)$.

Proof. We describe an $\left((1 / e)^{\left(\left(\frac{1}{2}-\epsilon\right) 2^{\lambda}-1\right)^{2} / 2^{2 \lambda-1}}, 2^{r}\left(q_{\mathrm{RO}}^{\mathrm{REncode}}+q_{\mathrm{RO}}^{\mathrm{Dec}}\right)+2^{\lambda}\right)-$ finder for $\Pi^{*}$.

Finder $\mathrm{F}^{\mathrm{RO}}\left(1^{\lambda}\right)$ :

1. Compute for any $c_{0}$ the set $\mathcal{E}\left(c_{0}\right)=\left\{c_{1} \mid \exists \rho:\left(c_{0}, c_{1}\right)=\Pi\right.$.Enc $\left.{ }^{\mathrm{RO}}\left(1^{\lambda} ; \rho\right)\right\}$;

2. Compute for any $c_{0}$ the set $\mathcal{M}\left(c_{0}\right)=\left\{\Pi\right.$.Dec $\left.\left(c_{0}, c_{1}\right) \mid c_{1} \in \mathcal{E}\left(c_{0}\right)\right\}$;

3. Find $c_{0}^{*}$ such that $\left|\mathcal{M}\left(c_{0}^{*}\right)\right|=\max _{c_{0}}\left|\mathcal{M}\left(c_{0}\right)\right|$;

4. Find $c_{1}^{(1)}, c_{1}^{(2)} \in \mathcal{E}\left(c_{0}^{*}\right)$ such that $\operatorname{RO}\left(c_{1}^{(1)}\right)=\operatorname{RO}\left(c_{2}^{(2)}\right)$ and $\Pi \cdot \operatorname{Dec}\left(c_{0}^{*}, c_{1}^{(1)}\right) \neq$ $\Pi . \operatorname{Dec}\left(c_{0}^{*}, c_{1}^{(2)}\right)$.

5. If such tuple does not exist output $\perp$, else output $\left(\bar{c}_{0}, \bar{c}_{1}, \bar{c}_{0}^{\prime}, \bar{c}_{1}^{\prime}\right)$ such that:

$$
\bar{c}_{0}=\bar{c}_{0}^{\prime}:=\left(c_{0}^{*}, \mathrm{RO}\left(c_{1}^{(1)}\right)\right) \quad \bar{c}_{1}:=\left(c_{1}^{(1)}, \mathrm{RO}\left(c_{0}^{*}\right)\right) \quad \bar{c}_{1}^{\prime}:=\left(c_{1}^{(2)}, \mathrm{RO}\left(c_{0}^{*}\right)\right) .
$$

We analyzes the probability that the finder outputs a valid triplet. 
Claim. For any $\epsilon(\lambda) \in \mathbb{R}, \ell(\lambda) \in \mathbb{N}$, if $\Pi$ is a $\left(\epsilon, \ell, 2^{r}\left(q_{\mathrm{RO}}^{\mathrm{REn} \text { code }}+q_{\mathrm{RO}}^{\mathrm{Dec}}\right)\right)$ noisy-leakage resilient randomness encoder then $\left|\mathcal{M}\left(c_{0}^{*}\right)\right| \geq\left(\frac{1}{2}-\epsilon\right) 2^{\lambda}$.

Proof (of the Claim). Suppose that for any $c_{0}$ we have $\left|\mathcal{M}\left(c_{0}\right)\right|<\left(\frac{1}{2}-\epsilon\right) 2^{\lambda}$. Consider the following attacker against noisy-leakage resilience.

Adversary $\mathrm{B}\left(\kappa_{0}, \kappa_{1}\right)$ :

1. Send the leakage function that on input $c_{0}$ outputs:

-1 if $\kappa_{1} \in \mathcal{M}\left(c_{0}\right)$ but $\kappa_{0} \notin \mathcal{M}\left(c_{0}\right)$,

- 0 if $\kappa_{0} \in \mathcal{M}\left(c_{0}\right)$ but $\kappa_{1} \notin \mathcal{M}\left(c_{0}\right)$,

$-\perp$ if $\kappa_{0} \in \mathcal{M}\left(c_{0}\right)$ and $\kappa_{1} \in \mathcal{M}\left(c_{0}\right)$.

let $b^{\prime}$ be the output of the leakage function;

2. If $b^{\prime}=\perp$ output a random bit, else output $b^{\prime}$.

Let $b$ be the challenge bit, the probability of $\kappa_{1-b} \in \mathcal{M}\left(c_{0}\right)$ is strictly smaller than $\left(\frac{1}{2}-\epsilon\right) 2^{\lambda} / 2^{\lambda}$. Notice that $\kappa_{b} \in \mathcal{M}\left(c_{0}\right)$, thus the adversary $B$ successfully guesses the challenge bit whenever the output of the second leakage function is not $\perp$. We can conclude that the advantage of $B$ is strictly greater then $\epsilon$.

By the claim above there exists at least $\left(\frac{1}{2}-\epsilon\right) 2^{\lambda}$ different values $c_{1}$ that decodes correctly with $c_{0}^{*}$ and whose decoded messages are pairwise different. Thus applying the birthday-paradox bound the probability that the finder successfully outputs a valid tuple is at least $(1 / e)^{\left(\left(\frac{1}{2}-\epsilon\right) 2^{\lambda}-1\right)^{2} / 2^{2 \lambda-1}}$. Finally, notice that the number of random oracle queries made by $F$ are:

$-2^{r}\left(q_{\mathrm{RO}}^{\mathrm{Enc}}+q_{\mathrm{RO}}^{\mathrm{Dec}}\right)$ to compute the sets $\mathcal{E}\left(c_{0}\right)$ for any $c_{0}$;

- At most $2^{\lambda}$ to compute the step 4 .

By applying Theorem 5 point 1 we have the statement of the theorem.

\section{References}

1. Divesh Aggarwal, Shashank Agrawal, Divya Gupta, Hemanta K. Maji, Omkant Pandey, and Manoj Prabhakaran. Optimal computational split-state non-malleable codes. In Eyal Kushilevitz and Tal Malkin, editors, TCC 2016-A, Part II, volume 9563 of $L N C S$, pages 393-417. Springer, Heidelberg, January 2016.

2. Divesh Aggarwal, Yevgeniy Dodis, and Shachar Lovett. Non-malleable codes from additive combinatorics. In ACM STOC, pages 774-783, 2014.

3. Divesh Aggarwal, Stefan Dziembowski, Tomasz Kazana, and Maciej Obremski. Leakage-resilient non-malleable codes. In Yevgeniy Dodis and Jesper Buus Nielsen, editors, TCC 2015, Part I, volume 9014 of LNCS, pages 398-426. Springer, Heidelberg, March 2015. 
4. Shashank Agrawal, Divya Gupta, Hemanta K. Maji, Omkant Pandey, and Manoj Prabhakaran. Explicit non-malleable codes against bit-wise tampering and permutations. In CRYPTO, pages 538-557, 2015.

5. Joël Alwen, Yevgeniy Dodis, Moni Naor, Gil Segev, Shabsi Walfish, and Daniel Wichs. Public-key encryption in the bounded-retrieval model. In Henri Gilbert, editor, EUROCRYPT 2010, volume 6110 of LNCS, pages 113-134. Springer, Heidelberg, May / June 2010.

6. Giuseppe Ateniese, Antonio Faonio, and Seny Kamara. Leakage-resilient identification schemes from zero-knowledge proofs of storage. In Jens Groth, editor, 15th IMA International Conference on Cryptography and Coding, volume 9496 of LNCS, pages 311-328. Springer, Heidelberg, December 2015.

7. Giuseppe Ateniese, Antonio Faonio, Bernardo Magri, and Breno de Medeiros. Certified Bitcoins. In Ioana Boureanu, Philippe Owesarski, and Serge Vaudenay, editors, ACNS 14, volume 8479 of $L N C S$, pages 80-96. Springer, Heidelberg, June 2014 .

8. Gianluca Brian, Antonio Faonio, Maciej Obremski, Mark Simkin, and Daniele Venturi. Non-malleable secret sharing against bounded joint-tampering attacks in the plain model. In Daniele Micciancio and Thomas Ristenpart, editors, CRYPTO 2020, Part III, volume 12172 of LNCS, pages 127-155. Springer, Heidelberg, August 2020.

9. Gianluca Brian, Antonio Faonio, and Daniele Venturi. Continuously non-malleable secret sharing for general access structures. In Dennis Hofheinz and Alon Rosen, editors, TCC 2019, Part II, volume 11892 of LNCS, pages 211-232. Springer, Heidelberg, December 2019.

10. Gianluca Brian, Antonio Faonio, and Daniele Venturi. Continuously non-malleable secret sharing: Joint tampering, plain model and capacity. Cryptology ePrint Archive, Report 2021/1128, 2021. https://ia.cr/2021/1128.

11. Eshan Chattopadhyay, Vipul Goyal, and Xin Li. Non-malleable extractors and codes, with their many tampered extensions. In Daniel Wichs and Yishay Mansour, editors, 48th ACM STOC, pages 285-298. ACM Press, June 2016.

12. Mahdi Cheraghchi and Venkatesan Guruswami. Non-malleable coding against bitwise and split-state tampering. In TCC, pages 440-464, 2014.

13. Sandro Coretti, Yevgeniy Dodis, Björn Tackmann, and Daniele Venturi. Nonmalleable encryption: Simpler, shorter, stronger. In TCC, pages 306-335, 2016.

14. Sandro Coretti, Antonio Faonio, and Daniele Venturi. Rate-optimizing compilers for continuously non-malleable codes. In Robert H. Deng, Valérie Gauthier-Umaña, Martín Ochoa, and Moti Yung, editors, ACNS 19, volume 11464 of $L N C S$, pages 3-23. Springer, Heidelberg, June 2019.

15. Sandro Coretti, Ueli Maurer, Björn Tackmann, and Daniele Venturi. From singlebit to multi-bit public-key encryption via non-malleable codes. In TCC, 2015.

16. Dana Dachman-Soled and Mukul Kulkarni. Upper and lower bounds for continuous non-malleable codes. In Dongdai Lin and Kazue Sako, editors, PKC 2019, Part I, volume 11442 of LNCS, pages 519-548. Springer, Heidelberg, April 2019.

17. Dana Dachman-Soled, Feng-Hao Liu, Elaine Shi, and Hong-Sheng Zhou. Locally decodable and updatable non-malleable codes and their applications. In $T C C$, pages 427-450, 2015.

18. Francesco Davì, Stefan Dziembowski, and Daniele Venturi. Leakage-resilient storage. In Juan A. Garay and Roberto De Prisco, editors, SCN 10, volume 6280 of LNCS, pages 121-137. Springer, Heidelberg, September 2010. 
19. Yevgeniy Dodis, Allison B. Lewko, Brent Waters, and Daniel Wichs. Storing secrets on continually leaky devices. In Rafail Ostrovsky, editor, 52nd FOCS, pages 688697. IEEE Computer Society Press, October 2011.

20. Yevgeniy Dodis, Leonid Reyzin, and Adam Smith. Fuzzy extractors: How to generate strong keys from biometrics and other noisy data. In Christian Cachin and Jan Camenisch, editors, EUROCRYPT 2004, volume 3027 of LNCS, pages 523-540. Springer, Heidelberg, May 2004.

21. Stefan Dziembowski and Sebastian Faust. Leakage-resilient cryptography from the inner-product extractor. In Dong Hoon Lee and Xiaoyun Wang, editors, ASI$A C R Y P T$ 2011, volume 7073 of $L N C S$, pages 702-721. Springer, Heidelberg, December 2011.

22. Stefan Dziembowski, Tomasz Kazana, and Maciej Obremski. Non-malleable codes from two-source extractors. In CRYPTO, pages 239-257, 2013.

23. Stefan Dziembowski and Krzysztof Pietrzak. Intrusion-resilient secret sharing. In 48th FOCS, pages 227-237. IEEE Computer Society Press, October 2007.

24. Stefan Dziembowski, Krzysztof Pietrzak, and Daniel Wichs. Non-malleable codes. In Andrew Chi-Chih Yao, editor, ICS 2010, pages 434-452. Tsinghua University Press, January 2010.

25. Antonio Faonio. Practical continuously non-malleable randomness encoders in the random oracle model. Cryptology ePrint Archive. https://ia.cr/2021/1269.

26. Antonio Faonio. Efficient fully-leakage resilient one-more signature schemes. In Mitsuru Matsui, editor, CT-RSA 2019, volume 11405 of $L N C S$, pages 350-371. Springer, Heidelberg, March 2019.

27. Antonio Faonio and Jesper Buus Nielsen. Fully leakage-resilient codes. In Serge Fehr, editor, PKC 2017, Part I, volume 10174 of LNCS, pages 333-358. Springer, Heidelberg, March 2017.

28. Antonio Faonio and Jesper Buus Nielsen. Non-malleable codes with split-state refresh. In Serge Fehr, editor, PKC 2017, Part I, volume 10174 of LNCS, pages 279-309. Springer, Heidelberg, March 2017.

29. Antonio Faonio, Jesper Buus Nielsen, Mark Simkin, and Daniele Venturi. Continuously non-malleable codes with split-state refresh. In Bart Preneel and Frederik Vercauteren, editors, ACNS 18, volume 10892 of $L N C S$, pages 121-139. Springer, Heidelberg, July 2018.

30. Antonio Faonio, Jesper Buus Nielsen, and Daniele Venturi. Mind your coins: Fully leakage-resilient signatures with graceful degradation. In Magnús M. Halldórsson, Kazuo Iwama, Naoki Kobayashi, and Bettina Speckmann, editors, ICALP 2015, Part I, volume 9134 of LNCS, pages 456-468. Springer, Heidelberg, July 2015.

31. Antonio Faonio and Daniele Venturi. Efficient public-key cryptography with bounded leakage and tamper resilience. In Jung Hee Cheon and Tsuyoshi Takagi, editors, ASIACRYPT 2016, Part I, volume 10031 of LNCS, pages 877-907. Springer, Heidelberg, December 2016.

32. Antonio Faonio and Daniele Venturi. Non-malleable secret sharing in the computational setting: Adaptive tampering, noisy-leakage resilience, and improved rate. In Alexandra Boldyreva and Daniele Micciancio, editors, CRYPTO 2019, Part II, volume 11693 of LNCS, pages 448-479. Springer, Heidelberg, August 2019.

33. Sebastian Faust, Pratyay Mukherjee, Jesper Buus Nielsen, and Daniele Venturi. Continuous non-malleable codes. In Yehuda Lindell, editor, TCC 2014, volume 8349 of LNCS, pages 465-488. Springer, Heidelberg, February 2014.

34. Serge Fehr, Pierre Karpman, and Bart Mennink. Short non-malleable codes from related-key secure block ciphers. IACR Trans. Symm. Cryptol., 2018(1):336-352, 2018 . 
35. Rosario Gennaro, Anna Lysyanskaya, Tal Malkin, Silvio Micali, and Tal Rabin. Algorithmic tamper-proof (ATP) security: Theoretical foundations for security against hardware tampering. In TCC, pages 258-277, 2004.

36. Bhavana Kanukurthi, Sai Lakshmi Bhavana Obbattu, and Sruthi Sekar. Nonmalleable randomness encoders and their applications. In Jesper Buus Nielsen and Vincent Rijmen, editors, EUROCRYPT 2018, Part III, volume 10822 of LNCS, pages 589-617. Springer, Heidelberg, April / May 2018.

37. Aggelos Kiayias, Feng-Hao Liu, and Yiannis Tselekounis. Practical non-malleable codes from l-more extractable hash functions. In Edgar R. Weippl, Stefan Katzenbeisser, Christopher Kruegel, Andrew C. Myers, and Shai Halevi, editors, ACM CCS 2016, pages 1317-1328. ACM Press, October 2016.

38. Xin Li. Improved non-malleable extractors, non-malleable codes and independent source extractors. In ACM STOC, pages 1144-1156, 2017.

39. Xin Li. Improved non-malleable extractors, non-malleable codes and independent source extractors. In Hamed Hatami, Pierre McKenzie, and Valerie King, editors, 49th ACM STOC, pages 1144-1156. ACM Press, June 2017.

40. Feng-Hao Liu and Anna Lysyanskaya. Tamper and leakage resilience in the splitstate model. In CRYPTO, pages 517-532, 2012.

41. Rafail Ostrovsky, Giuseppe Persiano, Daniele Venturi, and Ivan Visconti. Continuously non-malleable codes in the split-state model from minimal assumptions. In Hovav Shacham and Alexandra Boldyreva, editors, CRYPTO 2018, Part III, volume 10993 of LNCS, pages 608-639. Springer, Heidelberg, August 2018. 